ARTICLE

https://doi.org/10.1038/s41467-021-22867-w

\title{
Single-component multilayered self-assembling nanoparticles presenting rationally designed glycoprotein trimers as Ebola virus vaccines
}

Linling He ${ }^{1,4}$, Anshul Chaudhary ${ }^{1,4}$, Xiaohe Lin ${ }^{1,4}$, Cindy Sou', Tanwee Alkutkar', Sonu Kumar (i) 1, Timothy Ngo ${ }^{1}$, Ezra Kosviner ${ }^{1}$, Gabriel Ozorowski (1) ${ }^{1}$, Robyn L. Stanfield ${ }^{1}$, Andrew B. Ward (1) ${ }^{1}$, Ian A. Wilson (1) ${ }^{1,2 \bowtie} \&$ Jiang Zhu (iD) 1,3凶

Ebola virus (EBOV) glycoprotein (GP) can be recognized by neutralizing antibodies (NAbs) and is the main target for vaccine design. Here, we first investigate the contribution of the stalk and heptad repeat $1-\mathrm{C}\left(\mathrm{HR} 1_{\mathrm{C}}\right)$ regions to GP metastability. Specific stalk and HR1 $\mathrm{C}$ modifications in a mucin-deleted form (GP $\Delta$ muc) increase trimer yield, whereas alterations of $\mathrm{HR} 1_{\mathrm{C}}$ exert a more complex effect on thermostability. Crystal structures are determined to validate two rationally designed GP $\Delta$ muc trimers in their unliganded state. We then display a modified GPAmuc trimer on reengineered protein nanoparticles that encapsulate a layer of locking domains (LD) and a cluster of helper T-cell epitopes. In mice and rabbits, GP trimers and nanoparticles elicit cross-ebolavirus NAbs, as well as non-NAbs that enhance pseudovirus infection. Repertoire sequencing reveals quantitative profiles of vaccine-induced B-cell responses. This study demonstrates a promising vaccine strategy for filoviruses, such as EBOV, based on GP stabilization and nanoparticle display.

\footnotetext{
${ }^{1}$ Department of Integrative Structural and Computational Biology, The Scripps Research Institute, La Jolla, CA, USA. ${ }^{2}$ Skaggs Institute for Chemical Biology, The Scripps Research Institute, La Jolla, CA, USA. ${ }^{3}$ Department of Immunology and Microbiology, The Scripps Research Institute, La Jolla, CA, USA. ${ }^{4}$ These authors contributed equally: Linling He, Anshul Chaudhary, Xiaohe Lin. ${ }^{凶}$ email: wilson@scripps.edu; jiang@scripps.edu
} 
E bola virus (EBOV), a member of the Ebolavirus genus in the Filoviridae family ${ }^{1}$, can cause a severe human disease known as viral hemorrhagic fever ${ }^{2,3}$. EBOV was solely responsible for the largest filovirus outbreak in history in 2013-2016 that caused 11,325 deaths ${ }^{4}$. The EBOV outbreak in 2019 led to 2103 deaths $^{5}$ and was declared an international emergency on July 17, 2019, by the World Health Organization (WHO). In recent years, significant progress has been made to counter this deadly virus. Neutralizing antibodies (NAbs) have provided effective therapeutics for EBOV infection ${ }^{6-9}$, as demonstrated by the ZMapp cocktail of murine chimeric antibodies $^{10,11}$, as well as human antibodies ${ }^{12,13}$. Vaccines based on different delivery systems have been tested in humans ${ }^{14-17}$, of which rVSV-ZEBOV $\left(\right.$ Ervebo $\left.^{\circledR}\right)$-a replication-competent recombinant vesicular stomatitis virus (VSV) expressing a Zaire EBOV glycoprotein (GP) ${ }^{18-21}$ - was recently approved by the U. S. Food and Drug Administration (FDA) for human use. However, GP-specific antibody titers did not noticeably increase 7 days after rVSV-ZEBOV vaccination in humans ${ }^{15,22}$, in contrast to prior findings in nonhuman primates ${ }^{23}$. Additionally, a recent study reported the neurotropism of $\mathrm{rVSV}-\mathrm{ZEBOV}$ that resulted in damage to the eye and brain in neonatal mice ${ }^{24}$. Antibody-dependent enhancement (ADE) of infection was also found for antibodies isolated from human survivors ${ }^{25}$, suggesting that weak or non-NAbs induced by a suboptimal vaccine may cause adverse effects. Currently, protein-based subunit vaccines are not available but may be needed to boost the NAb response in the rVSV-ZEBOV-vaccinated population, or as an alternative vaccine solution.

EBOV GP, a trimer of GP1-GP2 heterodimers responsible for cell entry ${ }^{26}$, is recognized by the humoral immune response during natural infection ${ }^{27-29}$. The outbreak in 2013-2016 led to an enduring campaign to identify and characterize NAbs for $\mathrm{EBOV}^{30}$ and other filoviruses, such as Marburg virus $(\mathrm{MARV})^{31-33}$. As a result, panels of NAbs were isolated from human survivors, vaccinated humans, and immunized animals ${ }^{12,34-39}$. Crystallography ${ }^{40-43}$ and electron microscopy $(\mathrm{EM})^{44-47}$ revealed multiple sites of vulnerability on EBOV GP. A systematic study of 171 monoclonal antibodies (mAbs) defined eight epitopes ${ }^{48}$, six of which can be recognized by broadly neutralizing antibodies (bNAbs) ${ }^{9}$. Meanwhile, over the last decade, HIV-1 vaccine research has been driven largely by a strategy that focuses on bNAb isolation, the structural analysis of bNAbenvelope glycoprotein (Env) interactions, and immunogen design ${ }^{49,50}$. An important milestone in recent HIV-1 research was the development of native-like Env trimers, which have emerged as a promising vaccine platform ${ }^{51,52}$. In contrast to the growing number of EBOV (b)NAbs and their structures with GP, little attention has been given to the rational design of EBOV GP. As class-I viral fusion proteins ${ }^{53,54}$, HIV-1 Env and EBOV GP are inherently metastable, which is a property that has been studied in depth for HIV-1 Env ${ }^{55-57}$ but not yet for EBOV GP. Another advance in the HIV-1 vaccine field was to display Env trimers on self-assembling nanoparticles (NPs) ${ }^{58,59}$. Recombinant virus-like particles (VLPs) can protect against EBOV challenge in animals ${ }^{60-62}$, but manufacturing difficulties have hindered their development as human vaccines ${ }^{63}$. Therefore, the multivalent display of stabilized EBOV GP trimers on protein NPs may provide a promising solution for developing VLP-type subunit vaccines, but this possibility has yet to be explored.

Here, we investigated the causes of EBOV GP metastability and designed multilayered NP immunogens for in vivo evaluation. To facilitate GP purification, we developed an immunoaffinity column based on $\mathrm{mAb} 100^{12,42}$, which is specific to native-like, trimeric GP. We first examined the contribution of two regions in $\mathrm{GP} 2$, namely the stalk and heptad repeat $1-\mathrm{C}\left(\mathrm{HR} 1_{\mathrm{C}}\right)$ regions, to
GP metastability in a mucin-deleted Zaire EBOV GP construct (GP $\Delta$ muc). We extended the soluble GP ectodomain ( $\left.\mathrm{GP}_{\mathrm{ECTO}}\right)$ in the stalk region from residue 632 (C terminus of HR2) to 637 and introduced a W615L mutation based on a comparison of EBOV and MARV GPs. We also assessed eight proline mutations in $\mathrm{HR} 1_{\mathrm{C}}$, a region equivalent to the HR1 bend that is essential to HIV-1 Env metastability ${ }^{55-57}$, for their ability to prevent the GP transition from pre- to postfusion states. Both the stalk and $\mathrm{HR} 1_{\mathrm{C}}$-proline mutations increased trimer yield, whereas the latter exhibited a complex effect on GP thermostability. In addition, newly engineered inter-protomer disulfide bonds (SS) were tested for their ability to increase trimer stability. Crystal structures were solved for two redesigned GP $\Delta$ muc trimer constructs to validate the stalk and $H R 1_{C}$-proline mutations at the atomic level. We then displayed a redesigned GP $\Delta$ muc trimer on ferritin (FR), E2p, and I3-01 NPs. Locking domains (LDs) and helper T-cell epitopes were incorporated into E2p and I3-01 60-mers ${ }^{55,64}$ to stabilize the NP shell from the inside and create multilayered NP carriers. In mice and rabbits, GP trimer and NP vaccines induced distinct antibody responses, with enhanced pseudoviral infection observed for some constructs. The next-generation sequencing (NGS) analysis of GP-specific B cells revealed different patterns for NPs that present large trimeric spikes versus smaller antigens, such as hepatitis $\mathrm{C}$ virus (HCV) E2 core ${ }^{65}$. Our study thus reports on critical factors for EBOV GP metastability, single-component multilayered self-assembling protein NPs for the development of VLP-type vaccines, and a subunit vaccine strategy that is applicable to other filoviruses.

\section{Results}

Tag-free immunoaffinity purification of EBOV GP trimers. EBOV GP contains a heavily glycosylated mucin-like domain (MLD) that shields the glycan cap and neutralizing epitopes in GP1 and GP2 (Fig. 1a). A soluble, mucin-deleted form of Zaire EBOV GP (GP $\triangle$ muc) that produced the first ${ }^{40}$ and subsequent high-resolution ${ }^{66}$ crystal structures was used as a basic construct to investigate GP metastability (Fig. 1a). In HIV-1 vaccine research, immunoaffinity chromatography (IAC) columns based on bNAbs 2 G12 and PGT145 $5^{67,68}$ have been widely used to purify native-like Env trimers. 2G12 targets a glycan patch on a single gp120, whereas PGT145 binds the trimer apex and can separate closed trimers from partially open and misfolded Envs. These two bNAb columns have also been used to purify HIV-1 gp140-presenting $\mathrm{NPs}^{55,64,69}$. Likewise, GP-specific IAC columns may provide useful tools for EBOV vaccine research. Corti et al. recently identified two potent NAbs, mAb114 and mAb100, from a human survivor ${ }^{12}$. Misasi et al. elucidated the structural basis for neutralization by mAb114, which targets the receptor-binding site (RBS), and mAb100, which interacts with the GP1/GP2 interface and internal fusion loop (IFL) of two GP protomers ${ }^{42}$. Here, we examined the utility of mAb114 and mAb100 in IAC columns. The GP $\Delta$ muc constructs with and without a C-terminal foldon motif were transiently expressed in $250 \mathrm{ml}$ HEK293F cells and purified on an antibody column prior to size-exclusion chromatography (SEC) using a Superdex 200 10/300 GL column and blue native polyacrylamide gel electrophoresis (BN-PAGE). With mAb114, both GP $\Delta$ muc samples showed aggregate $(\sim 9 \mathrm{ml})$, dimer $(\sim 12 \mathrm{ml})$, and monomer $(\sim 13.5-14 \mathrm{ml})$ peaks in the SEC profiles, but only GP $\Delta$ muc-foldon showed a visible trimer peak $(\sim 10.5-11 \mathrm{ml})$ in SEC and a band of slightly less than $440 \mathrm{kD}$ on the $\mathrm{BN}$ gel (Fig. 1b). Following mAb100 purification, GP $\Delta$ muc produced a low overall yield, whereas GP $\Delta$ muc-foldon demonstrated high trimer purity without any monomer or dimer peaks. Consistently, GP $\Delta$ muc-foldon registered a single trimer band on the BN gel (Fig. 1c). Altogether, both mAb114 and mAb100 can 
a

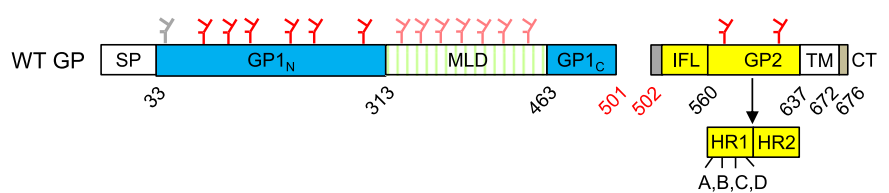

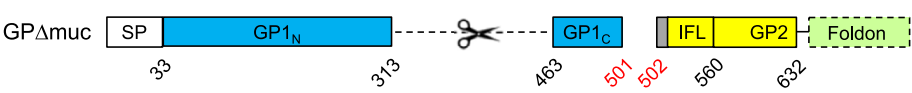

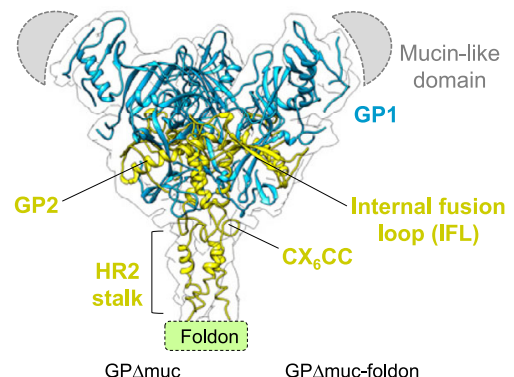

b

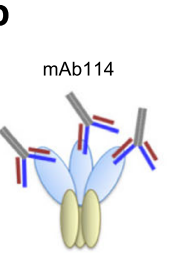
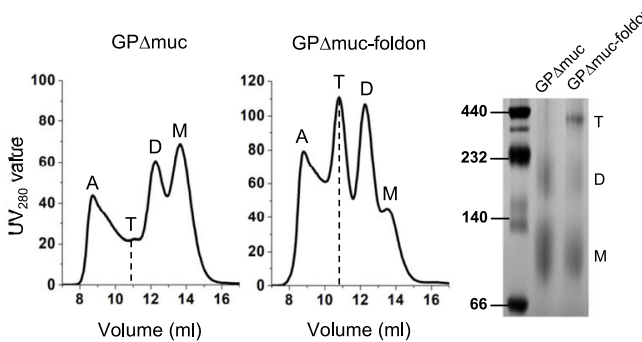

C
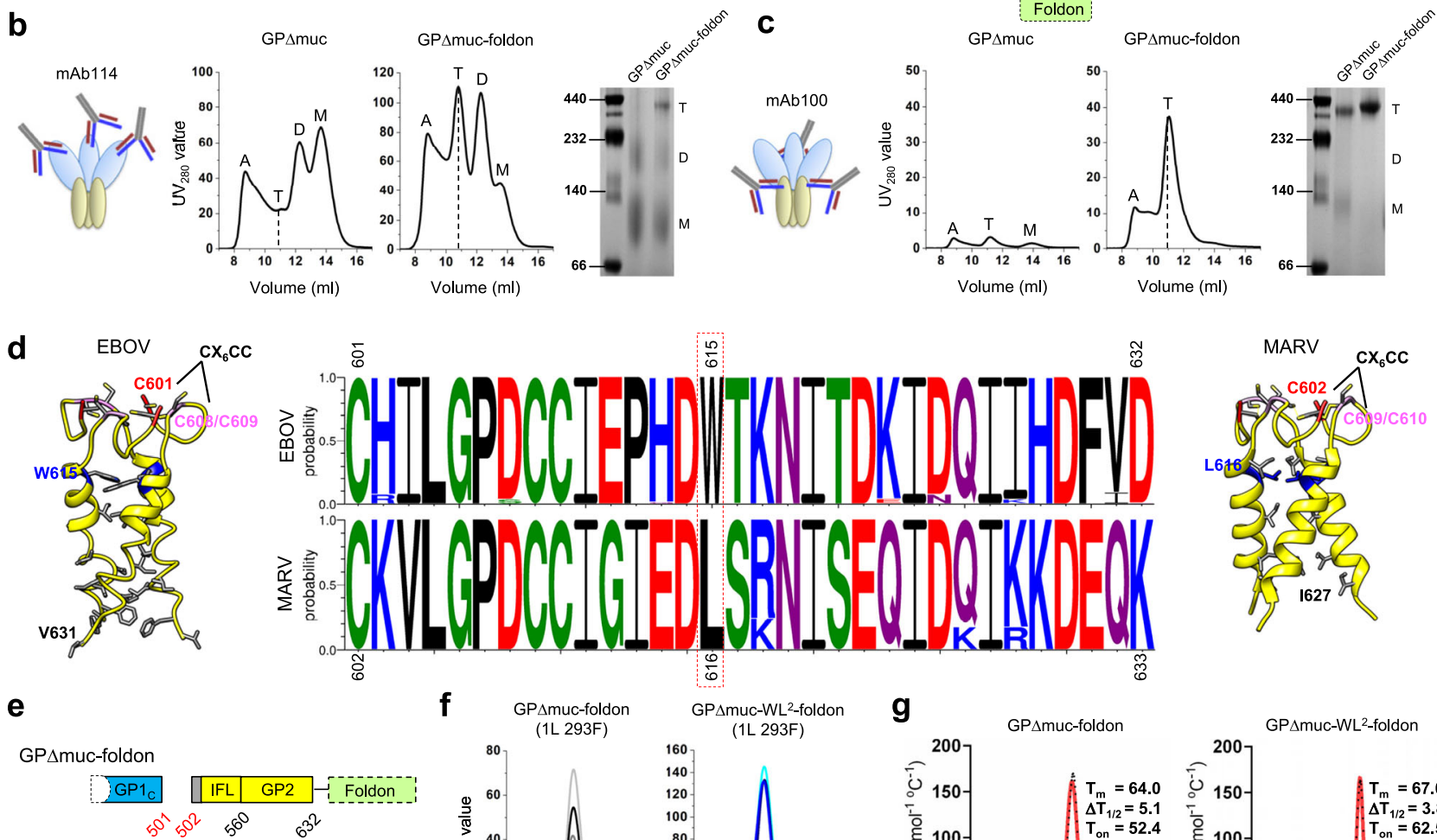

GPAmuc-W615L-L(or WL2)-foldon

GP1C IFL $\mid$ GP2 $10 .-10$

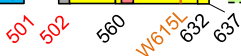
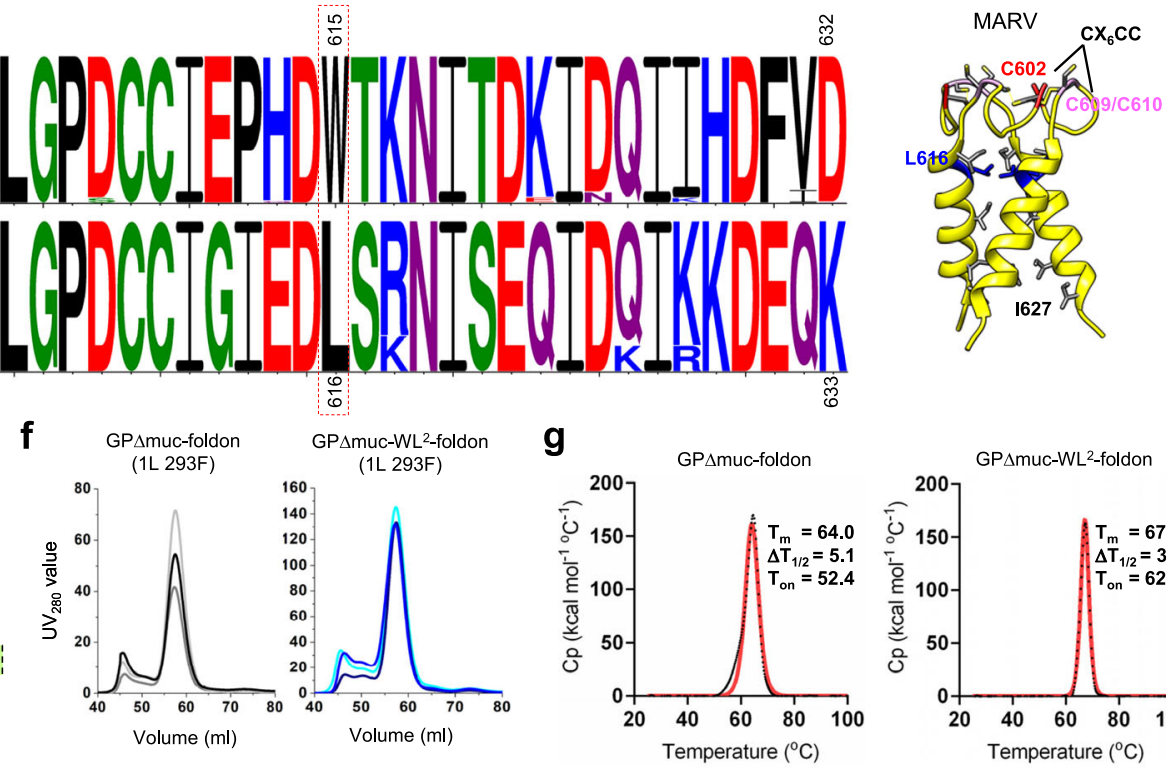

h

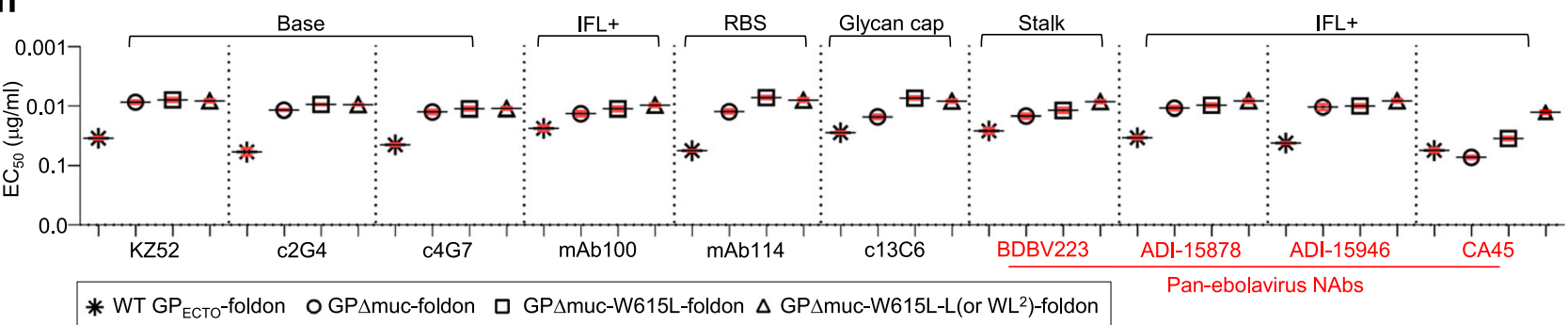

Fig. 1 Design and characterization of EBOV GPAmuc trimers with modified HR2 stalk. a Left: Schematic representation of EBOV GP and GP $\triangle$ muc. GP1 $\mathrm{N} / \mathrm{C}$ termini $\left(\mathrm{GP} 1_{\mathrm{N} / \mathrm{C}}\right.$ ), mucin-like domain (MLD), internal fusion loop (IFL), heptad repeat regions 1/2 (HR1/HR2), transmembrane region (TM), and cytoplasmic tail (CT) are labeled with $N$-linked glycans, which are indicated as gray (mutated), red, and pink (within MLD) branches. Right: Ribbon representation of EBOV GP (PDB: 5JQ3) in transparent molecular surface, with GP1 in dodger blue and GP2 in yellow. The MLD and foldon are shown as a gray half oval and a green rectangle, respectively. $\mathbf{b}$ Schematic representation of mAb114 bound to EBOV GP (left), SEC profiles of mAb114-purified GP $\Delta$ muc and GP $\Delta$ muc-foldon (middle) from a Superdex 200 10/300 column, and BN-PAGE gel (right). c Schematic representation of mAb100 bound to EBOV GP (left), SEC profiles of mAb100-purified GP $\Delta$ muc and GP $\Delta$ muc-foldon (middle) from a Superdex 200 10/300 column, and BN-PAGE gel (right). In $\mathbf{b}$, c, GP species (A aggregate, $T$ trimer, D dimer, $M$ monomer) are labeled on the SEC profile and BN-PAGE gel. $\mathbf{d}$ Ribbon representation of EBOV HR2 stalk (left) and MARV HR2 stalk (right) with $\mathrm{CX}_{6} \mathrm{CC}$ motif and residues of interest indicated. Middle: Logo analysis of EBOV and MARV HR2 sequences. e Schematic representation of GP $\Delta$ muc-W615L-L (or WL2²)-foldon. f SEC profiles of 293F-expressed, mAb100-purified GP $\Delta$ muc-foldon and GP $\Delta$ muc-WL2-foldon from a HiLoad Superdex 200 16/600 column for three production runs. $\mathbf{g}$ Thermostability of GP $\Delta$ muc-foldon and GP $\Delta$ mucWL2-foldon, with $T_{m}, \Delta T_{1 / 2}$, and $T_{\text {on }}$ measured by DSC. $\mathbf{h}$ EC 50 ( $\left.\mu \mathrm{g} / \mathrm{ml}\right)$ values of EBOV GP-foldon, GP $\Delta$ muc-foldon, GP $\Delta$ muc-W615L-foldon, and $\mathrm{GP} \Delta$ muc-WL ${ }^{2}$-foldon binding to 10 representative antibodies. Four pan-ebolavirus NAbs are colored in red. Antibody binding was measured by ELISA in duplicates, with mean value and standard deviation (SD) shown as black and red lines, respectively. Source data are provided as a Source Data file. 
be used to purify EBOV GP, but mAb100 offers a more effective IAC method for purifying native-like trimers due to its recognition of a quaternary epitope. The stark difference in trimer yield between the two GP $\Delta$ muc constructs after mAb100 purification also suggests a strong tendency for trimer dissociation without the C-terminal foldon motif.

Effect of HR2 stalk on EBOV GP metastability. Previously, we demonstrated that the gp41 ectodomain (gp41 $1_{\mathrm{ECTO}}$ ) is the source of HIV-1 Env metastability ${ }^{55}$. Unlike HIV-1 Env ${ }^{70,71}$, in which the gp41 HR2 helix is packed against the bottom of the gp41 HR1 helix and gp120 C1/C5 loops and forms extensive interactions at the gp140 trimer base ${ }^{70,71}$, EBOV GP has a long, extended HR2 stalk (Fig. 1a, right). Even in the high-resolution GP $\Delta$ muc structures ${ }^{66,72,73}$, the HR2 stalk still contains less helical content than most coiled-coils in the Protein Data Bank (PDB), $\sim 15$ versus $\sim 30$ aa, and becomes less regular and unwound toward the $\mathrm{C}$ terminus, suggesting an inherent instability in HR2 (Fig. 1d, left). Recently, King et al. solved a $3.17 \AA$ Å-resolution structure for MARV GP $\triangle$ muc bound to a human mAb, MR191 ${ }^{32}$. Surprisingly, the MARV HR2 stalk adopted a well-formed coiled-coil with canonical sidechain packing along the threefold axis (Fig. 1d, right). To identify the cause of this difference in HR2, we obtained EBOV and MARV GP sequences from the Virus Pathogen Database and Analysis Resource (ViPR, https://www. viprbrc.org). A total of 274 EBOV GPs and 87 MARV GPs were used for sequence conservation analysis of the region spanning the $\mathrm{CX}_{6} \mathrm{CC}$ motif and HR2 stalk, residues 601-632 for EBOV and residues 602-633 for MARV, respectively (Fig. 1d, middle). Most inward-facing amino acids were conserved except for W615 in EBOV and the equivalent L616 in MARV. Indeed, structural analysis revealed a critical difference at this position: the three W615s in EBOV GP (PDB: 5JQ3) formed a wide triangle at the neck of the coiled-coil with a C $\alpha$ distance of $11.1 \AA$ and $C \beta$ distance of $9.0 \AA$; in contrast, with the smaller and more hydrophobic L616, a C $\alpha$ distance of $10.5 \AA$ and $C \beta$ distance of $8.3 \AA$ were observed in MARV GP (PDB: 6BP2). This finding suggests that a W615L mutation may improve the stability of EBOV GP.

To further examine the effect of the HR2 stalk on GP trimer stability, we created three GP $\Delta$ muc constructs by replacing residues 617-632 with the coiled-coil from a GCN4 leucine zipper (PDB: 2WPZ, residues 3-33) and by extending the $\mathrm{C}$ terminus to D637 and N643 to include a newly identified bNAb epitope ${ }^{74}$ that spans HR2 and the membrane-proximal external region (MPER), termed "L" and "Ext", respectively (Fig. S1a). Notably, D637 is also important for proteolytic cleavage by tumor necrosis factor $\alpha$-converting enzyme, which enables GP to be shed from the virus surface $^{75}$. These three constructs were characterized by SEC and BN-PAGE following transient expression in 250-ml HEK293F cells and purification on an antibody column. With mAb114 purification, all three HR2 stalk variants showed a greater trimer yield than wildtype GP $\triangle$ muc in SEC (Fig. S1b), with trimer bands observed only for the stalk variants on the BN gel (Fig. S1c, left). Upon mAb100 purification, all three HR2 stalk variants showed more visible trimer bands than wildtype GP $\Delta$ muc on the $B N$ gel (Fig. S1c, right). Of the three designs, "2WPZ" improved GP stability at the cost of replacing the entire HR2 stalk in GP2 but provided supporting evidence for the W615L mutation, which presumably increases coiled-coil content in the HR2 stalk (Fig. 1d). Overall, the "L" extension appeared to be a more practical solution as it modestly improved trimer stability with the inclusion of a recently identified bNAb epitope ${ }^{74}$.

We next combined the W615L mutation and "L" extension in a single construct named GP $\Delta$ muc-W615L-L-foldon, or simply GP $\Delta$ muc-WL ${ }^{2}$-foldon (Fig. 1e). This construct, along with
GP $\Delta$ muc-foldon, was transiently expressed in 1-L HEK293F cells and purified by an mAb100 column prior to SEC on a HiLoad Superdex 200 16/600 GL column (Fig. 1f). In three production runs, GP $\Delta$ muc-WL ${ }^{2}$-foldon consistently outperformed the wildtype construct, showing a twofold higher trimer peak in the SEC profile and a $\sim 2.6$-fold greater trimer yield after SEC $(1.3 \mathrm{mg}$ versus $0.5 \mathrm{mg}$ ). Thermostability was assessed by differential scanning calorimetry (DSC) for two purified GP trimers (Fig. 1g). The thermal denaturation midpoint $\left(T_{\mathrm{m}}\right)$ value for the stalkstabilized trimer was $3^{\circ} \mathrm{C}$ higher than the wildtype trimer $\left(67^{\circ} \mathrm{C}\right.$ versus $\left.64^{\circ} \mathrm{C}\right)$. Stalk stabilization also increased the onset temperature $\left(T_{\text {on }}\right)$ from 52.4 to $62.5^{\circ} \mathrm{C}$, with a narrower half width of the peak $\left(\Delta T_{1 / 2}\right)$ than the wildtype trimer $\left(3.8^{\circ} \mathrm{C}\right.$ versus $5.1^{\circ} \mathrm{C}$ ). Antigenicity was assessed for four mAb100/SEC-purified EBOV GP trimers in the enzyme-linked immunosorbent assay (ELISA) (Fig. $1 \mathrm{~h}$ and Fig. S1d, e). Ten antibodies were tested, including three NAbs targeting the base $\left(\mathrm{KZ}^{2} 2^{76}, \mathrm{c} 2 \mathrm{G} 4\right.$, and $c 4 \mathrm{G}^{10}$ ), two human $\mathrm{NAbs}^{12}-\mathrm{mAb} 100$ (IFL) and mAb114 (RBS), a non-NAb directed to the glycan cap $\left(\mathrm{c}^{3} \mathrm{C} 6^{10}\right)$, and four panebolavirus bNAbs targeting the conserved HR2-MPER epitope $\left(\mathrm{BDBV} 223^{74}\right)$ and IFL (ADI-15878, ADI-15946 37,38 , and CA45 ${ }^{36,77}$ ). The GP $\Delta$ muc trimer showed notably improved antibody binding with respect to the $\mathrm{GP}_{\mathrm{ECTO}}$ trimer, with an up to 7.6-fold difference in the half maximal effective concentration $\left(\mathrm{EC}_{50}\right)$, indicating that the MLD can shield much of the GP from antibody recognition. The two stalk modifications only modestly increased recognition of the RBS, stalk, and IFL epitopes by their respective (b)NAbs except for CA45 $5^{36,77}$, for which the $\mathrm{WL}^{2}$ mutation led to a 5.6-fold change in $\mathrm{EC}_{50}$ compared with $\mathrm{GP} \Delta$ muc-foldon. A $50 \%$ reduction in $\mathrm{EC}_{50}$ observed for $\mathrm{GP} \Delta$ muc$\mathrm{WL}^{2}$-foldon binding to BDBV223 confirmed that the $\mathrm{L}$ extension into MPER can improve the recognition of this conserved bNAb epitope at HR2-MPER ${ }^{74}$. King et al. proposed two scenarios for ebolavirus GP to expose the BDBV223 epitope: one of the HR2 helices is splayed apart from the coiled-coil, or the whole GP is lifted or tilted with respect to the membrane ${ }^{74}$. It is perhaps more plausible that ebolavirus GP may adopt an open stalk conformation similar to the parainfluenza virus 5 fusion $(\mathrm{F})$ protein, which has a long HR-B stalk ${ }^{78}$. Altogether, $\mathrm{WL}^{2}$ considerably improved the trimer yield and thermostability for EBOV GP but only exerted a modest effect on antigenicity because the C-terminal foldon motif in these constructs could retain GP $\Delta$ muc in a trimeric form, which is required for (b)NAb binding.

Effect of the HR1 $1_{C}$ bend on EBOV GP metastability. Structures of the prefusion glycoprotein and the postfusion six-helix-bundle have been determined for HIV-1 Env ${ }^{70,71,79}$ and EBOV/MARV $\mathrm{GP}^{40,80-82}$. Previously, we identified the $\mathrm{N}$ terminus of HR1 $\left(\mathrm{HR} 1_{\mathrm{N}}\right.$, residues 547-569) as a major cause of HIV-1 Env metastability 56 because this region undergoes a drastic conformational change during fusion. We and others stabilized diverse HIV-1 Envs by replacing the 22 -aa HR1 $1_{\mathrm{N}}$ with an optimized 8-aa loop ${ }^{55-57,83}$. The equivalent region in EBOV GP (residues $576-583$ ), $\mathrm{HR}_{\mathrm{C}}{ }^{40}$, forms a $21-\AA$ triangular interior at around two-thirds of the height of the chalice that spans $94-\AA$ wide at the rim (Fig. 2a). This prefusion $\mathrm{HR} 1_{\mathrm{C}}$ bend will refold to two helical turns in the center of a long helix in the postfusion state (Fig. $2 \mathrm{~b}$, left). Here, we hypothesized that $\mathrm{HR} 1_{\mathrm{C}}$ is essential to EBOV GP metastability. Since $\mathrm{HR} 1_{\mathrm{C}}$ in wildtype EBOV GP is equivalent in length $(8 \mathrm{aa})$ to a truncated engineered $\mathrm{HR} 1_{\mathrm{N}}$ in the prefusion-optimized HIV-1 $\mathrm{Env}^{55,56}$, metastability in EBOV GP may not be as sensitive to $H R 1_{C}$ length and likely requires a different solution. We thus hypothesized that a proline mutation in one of the eight residues in $H R 1_{C}$ can rigidify the HR1 $1_{C}$ bend and improve EBOV GP trimer stability (Fig. 2b, right). Similar 
a

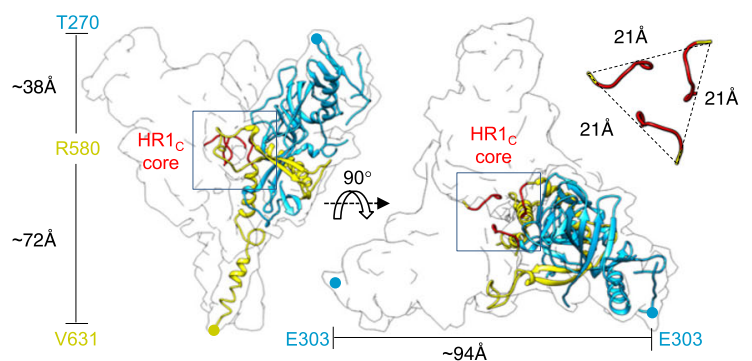

b

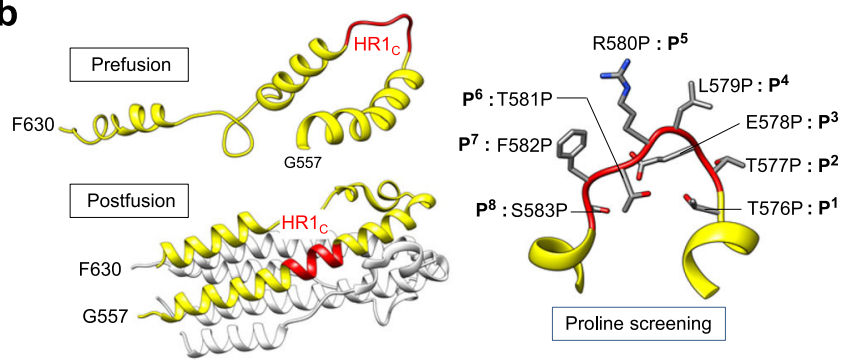

C

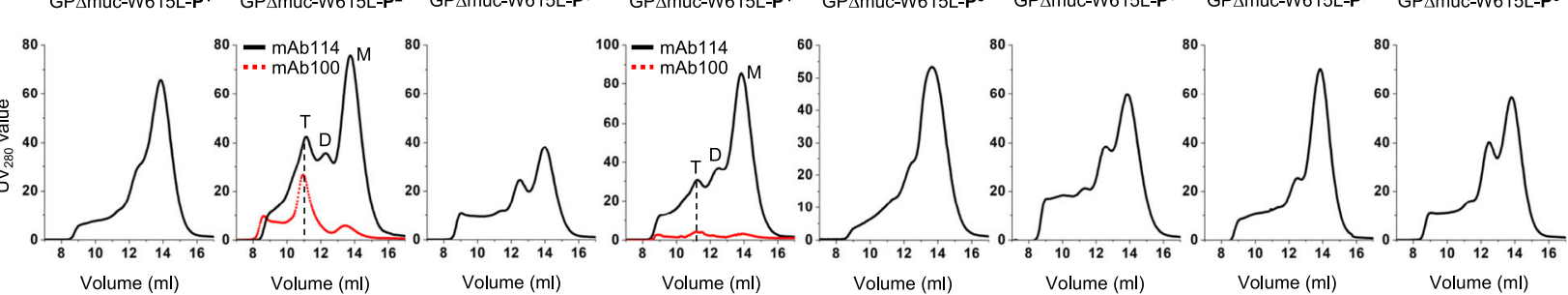

d

GPAmuc-W615L-Pn (n=1-8)

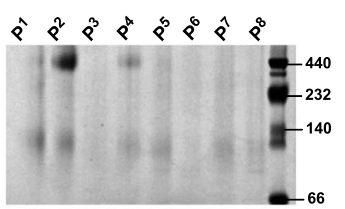

e

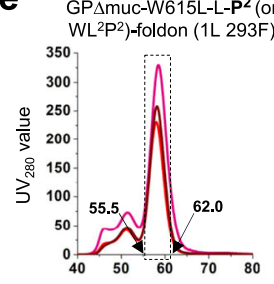

Volume $(\mathrm{ml})$ f

GP $\Delta$ muc-WL2P2-foldon
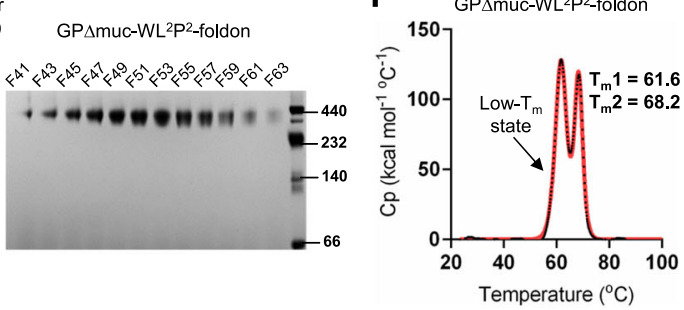

g

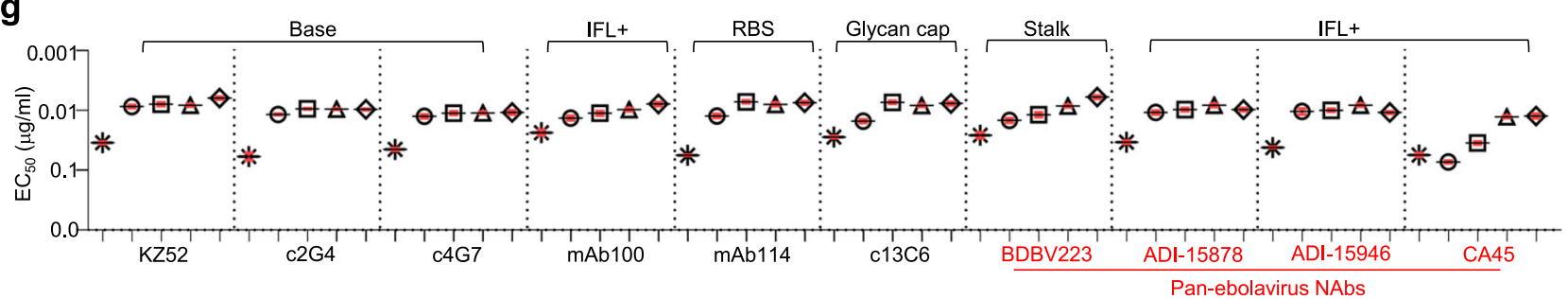

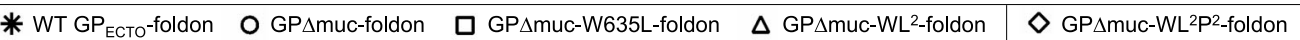

h

\begin{tabular}{|c|c|c|c|c|c|c|c|c|c|c|}
\hline \multicolumn{11}{|c|}{$K_{D}$ values for two EBOV GP $\Delta$ muc trimers binding to $10 \mathrm{lgG}$ antibodies $(\mathrm{nM})^{\text {a }}$} \\
\hline & KZ52 & c2G4 & c4G7 & mAb100 & mAb114 & $\mathrm{c} 13 \mathrm{C} 6$ & BDBV223 & $\mathrm{ADI}-15878$ & ADI-15946 & CA45 \\
\hline don & $<1.0 \mathrm{E}^{-3}$ & $<1.0 \mathrm{E}^{-3}$ & $<1.0 \mathrm{E}^{-3}$ & $<1.0 \mathrm{E}^{-3}$ & $<1.0 \mathrm{E}^{-3}$ & $<1.0 \mathrm{E}^{-3}$ & $<1.0 \mathrm{E}^{-3} \sim 0.21$ & $<1.0 \mathrm{E}^{-3}$ & $<1.0 \mathrm{E}^{-3}$ & $<1.0 \mathrm{E}^{-3}$ \\
\hline$G P \Delta m u c-W L^{2} P^{2}$-foldon & $<1.0 \mathrm{E}^{-3}$ & $<1.0 \mathrm{E}^{-3}$ & $<1.0 \mathrm{E}^{-3} \sim 0.19$ & $<1.0 \mathrm{E}^{-3}$ & $<1.0 \mathrm{E}^{-3}$ & $<1.0 \mathrm{E}^{-3}$ & $<1.0 \mathrm{E}^{-3} \sim 0.28$ & $<1.0 \mathrm{E}^{-3}$ & $<1.0 \mathrm{E}^{-3} \sim 27.9$ & $<1.0 \mathrm{E}^{-3} \sim 46.6$ \\
\hline
\end{tabular}

${ }^{a} K_{D}$ values from bio-layer interferometry (BLI) using six GP concentrations $(400-12.5 \mathrm{nM})$. Octet sensorgrams are shown in supplementary Fig. $\mathrm{S} 1 \mathrm{~h}$.

Fig. 2 Design and characterization of EBOV GPAmuc trimers with modified HR1 $\mathbf{C}_{\mathbf{c}}$ bend. a Ribbon representation of EBOV GP $\Delta$ muc protomer (PDB: 5JQ3) in transparent molecular surface with GP1 in dodger blue, GP2 in yellow, and HR1C bends from three protomers in red. Left: side view. Right: top view. b Left: Ribbon representation of the HR1 region in the prefusion (top, PDB ID: 5JQ3) and postfusion (bottom, PDB ID: 2EBO) states with the HR1 region in yellow and the HR1 $\mathrm{C}$ bend in red. Right: Zoomed-in view of the $\mathrm{HR} 1_{\mathrm{C}}$ bend with the eight residues in this region shown as sticks and labeled with the proline mutations, $P^{1}-P^{8}$. c SEC profiles of mAb114-purified GPAmuc-W615L-Pn variants ( $\left.n=1-8\right)$ from a Superdex 200 10/300 column. SEC profiles of mAb100purified GPAmuc-W615L-P2 and - $\mathrm{P}^{4}$ are shown in the red dotted line. Trimer (T), dimer (D), and monomer (M) peaks are labeled on the SEC profiles for $\mathrm{P}^{2}$ and $\mathrm{P}^{4}$, with the trimer peak marked with a black dashed line. $\mathbf{d}$ BN-PAGE gel of mAb114-purified GP $\Delta$ muc-W615L-Pn variants ( $n=1-8$ ). e SEC profiles of 293F-expressed, mAb100-purified GP $\Delta$ muc-W615L-L-P2 (or WL2 ${ }^{2}$ )-foldon from a HiLoad Superdex 200 16/600 column (left) and BN-PAGE gel of SEC fractions 41-63 (55.5-62.0 ml) (right). SEC profiles were from three production runs. $\mathbf{f}$ Thermostability of GP $\Delta m u c-W L^{2} \mathrm{P}^{2}-$ foldon and GP $\Delta$ muc-WL2P4 foldon, with $T \mathrm{~m}, \Delta T_{1 / 2}$, and $T_{\text {on }}$ measured by DSC. $\mathbf{g} \mathrm{EC}_{50}(\mu \mathrm{g} / \mathrm{ml})$ values of EBOV GP-foldon, GP $\Delta$ muc-foldon, GP $\Delta$ muc-W615L-foldon, GP $\Delta$ muc-WL2foldon, and GPAmuc-WL2 ${ }^{2}$-foldon binding to 10 representative antibodies. Four pan-ebolavirus NAbs are colored in red. Antibody binding was measured by ELISA in duplicates, with mean value and standard deviation (SD) shown as black and red lines, respectively. $\mathbf{h} K_{D}$ values of GP $\Delta$ Muc-foldon and GP $\Delta$ muc-WL2P2-foldon binding to 10 representative antibodies. BLI was performed on an Octet RED96 instrument using a trimer titration series of six concentrations (400-12.5 $\mathrm{nM}$ by twofold dilution) and kinetics (AHC) biosensors. The $K_{D}$ values were calculated using a global fit 2:1 model. Source data are provided as a Source Data file. 
proline mutations in $\mathrm{HR} 1_{\mathrm{N}}$ have effectively stabilized HIV-1 Env trimers ${ }^{56,68}$. To examine this possibility, eight GP $\Delta$ muc-W615L variants, each with a proline mutation in $\mathrm{HR}_{\mathrm{C}}$ (termed $\mathrm{P}^{1}$ to $\mathrm{P}^{8}$ ) but without the $\mathrm{L}$ extension and $\mathrm{C}$-terminal foldon motif, were characterized by SEC following 250-ml $293 \mathrm{~F}$ expression and IAC. After mAb114 purification, most proline mutations showed little effect on the distribution of GP species except for T577P $\left(\mathrm{P}^{2}\right)$ and L579P $\left(\mathrm{P}^{4}\right)$, which appeared to have a trimer peak at $\sim 11 \mathrm{ml}$ in their SEC profiles (Fig. 2c). After mAb100 purification, only $\mathrm{P}^{2}$ and $\mathrm{P}^{4}$ produced any trimer yield, with a higher SEC peak observed for $\mathrm{P}^{2}$ that corresponded to well-formed trimers (Fig. 2c). The mAb100-purified GP was analyzed by BN-PAGE, which showed a trimer band for $\mathrm{P}^{2}$ and $\mathrm{P}^{4}$ (Fig. 2d). Overall, the T577P mutation $\left(\mathrm{P}^{2}\right)$ can considerably increase trimer yield, whereas the L579P mutation $\left(\mathrm{P}^{4}\right)$ has a less pronounced effect.

Next, the T577P mutation $\left(\mathrm{P}^{2}\right)$ was incorporated into the $\mathrm{GP} \Delta$ muc-WL $\mathrm{WL}^{2}$-foldon construct, resulting in a new design named $\mathrm{GP} \Delta$ muc-WL $\mathrm{WL}^{2}$-foldon. This construct was transiently expressed in 1-L $293 \mathrm{~F}$ cells and purified using an mAb100 column for SEC characterization on a HiLoad Superdex 200 16/ $600 \mathrm{GL}$ column. In three production runs, GP $\Delta$ muc-WL $\mathrm{WL}^{2} \mathrm{P}^{2}$ foldon generated a trimer peak that was twofold and fourfold higher than GP $\Delta$ muc-WL ${ }^{2}$-foldon and wildtype GP $\Delta$ muc-foldon, respectively, with an average yield of $2.6 \mathrm{mg}$ after SEC (Fig. 2e, left). Protein collected in the range of 55.5-62.0 $\mathrm{ml}$ was analyzed by BN-PAGE, which displayed a trimer band across all fractions without any detectable impurity (Fig. 2e, right). The thermostability of GP $\Delta$ muc-WL $\mathrm{W}^{2} \mathrm{P}^{2}$-foldon was determined by DSC after mAb100 and SEC purification. Unexpectedly, two transition peaks were observed in the thermogram, one registered at a lower $T_{\mathrm{m}}$ of $61.6^{\circ} \mathrm{C}$ and the other at a higher $T_{\mathrm{m}}$ of $68.2^{\circ} \mathrm{C}$ (Fig. $2 \mathrm{f}$, left). To this end, a second construct containing the L579P mutation $\left(\mathrm{P}^{4}\right)$, termed GP $\Delta$ muc-WL ${ }^{2} \mathrm{P}^{4}$-foldon, was also assessed by DSC (Fig. 2f, right). Although only one peak was observed in the thermogram with a $T_{\mathrm{m}}$ of $67.0^{\circ} \mathrm{C}$, a slight widening at the onset of the peak suggested a similar unfolding behavior upon heating. Thus, DSC revealed the complexity associated with a prolinerigidified $H R 1_{C}$ bend, which may increase the trimer yield at the cost of reducing or modifying the GP thermostability profile. The antigenicity of GP $\Delta$ muc-WL ${ }^{2} \mathrm{P}^{2}$-foldon was first assessed by ELISA using the same panel of 10 antibodies (Fig. $2 \mathrm{~g}$ and Fig. S1f, g). GP $\Delta$ muc-WL ${ }^{2} \mathrm{P}^{2}$-foldon appeared to bind more favorably to bNAb BDBV223 than GPAmuc-WL ${ }^{2}$-foldon, with a twofold reduction in $\mathrm{EC}_{50}$. In the bio-layer interferometry (BLI) analysis (Fig. $2 \mathrm{~h}$ and Fig. S1h), the GP $\Delta$ muc-WL $\mathrm{WL}^{2} \mathrm{P}^{2}$-foldon trimer and wildtype GP $\Delta$ muc-foldon trimer yielded nearly indistinguishable kinetic profiles, with nano- to picomolar equilibrium dissociation constant $\left(K_{D}\right)$ values, consistent with the fast on-rates and slow off-rates in antibody binding.

Our results demonstrated the importance of $\mathrm{HR} 1_{\mathrm{C}}$ to $\mathrm{EBOV}$ GP metastability and the sensitivity of $H R 1_{C}$ to proline mutation. Recently, Rutten et al. tested proline mutations in $H R 1_{C}$ along with a K588F mutation to stabilize filovirus GP trimers ${ }^{84}$. A similar increase in trimer yield was observed for the T577P mutant, but the reported thermostability data appeared to be inconsistent with our DSC measurements. Further investigations are thus warranted to understand the role of $H R 1_{C}$ in filoviruscell fusion and GP stability. Nevertheless, the combined stalk/ $\mathrm{HR} 1_{\mathrm{C}}$ modification, $\mathrm{WL}^{2} \mathrm{P}^{2}$, was found to have no adverse effect on GP binding to diverse (b)NAbs.

GP stabilization with inter-protomer disulfide bonds. Engineered disulfide (SS) bonds have been used to stabilize viral glycoproteins, as demonstrated for HIV-1 $\mathrm{Env}^{68}$, respiratory syncytial virus $\mathrm{F}^{85}$, and Lassa virus GP complex (GPC) ${ }^{86}$. EBOV
GP already contains an endogenous SS bond linking GP1 and GP2 (C53-C609). Thus, we examined whether inter-protomer SS bonds can be used to stabilize trimer formation and lock the GP in a "closed" trimer. Based on a high-resolution EBOV GP $\triangle$ muc structure (PDB ID: 5JQ3), we identified inter-protomer amino acid pairs whose $C_{\beta}-C_{\beta}$ distances are within a cutoff of $4.72 \AA$ defined by Rosetta ${ }^{87}$ (Fig. S2a). Of the nine pairs that were identified, three were removed after visual inspection because they may interfere with an existing SS bond or a hydrophobic cluster. The remaining six pairs were divided into three groups: IFL-head group (SS1/2/4), IFL-NHR group (SS/5), and HR2 group (SS6) (Fig. S2b). Six GP $\Delta$ muc-SS constructs without Cterminal foldon were designed and characterized by SEC following transient expression in 250-ml $293 \mathrm{~F}$ cells and purification using an mAb114 column or mAb100 column (Fig. S2c). Diverse SEC profiles were observed for the mAb114-purified SS variants, with SS2 showing a notable trimer fraction. Upon mAb100 purification, only SS2, SS3, and SS5 resulted in a measurable protein yield, with SS2 showing a clean trimer peak without dimer/monomer species. The BN-PAGE analysis of mAb114 and mAb100-purified SS variants exhibited consistent patterns, with S22 showing a trimer band slightly below $440 \mathrm{kD}$ on the gel (Fig. S2d). However, the incorporation of SS2, SS3, or SS5 into the GP $\Delta$ muc-foldon construct led to abnormal SEC profiles regardless of the antibody column used for purification, suggesting their incompatibility with the foldon trimerization motif (Fig. S2e). To this end, antigenicity was assessed only for the mAb100/SECpurified GP $\Delta$ muc-SS2 trimer. In the ELISA, GP $\Delta$ muc-SS2 showed identical or improved antibody binding compared with $\mathrm{GP} \Delta$ muc-foldon, with a 3.7 -fold reduction in $\mathrm{EC}_{50}$ for bNAb CA45 (Fig. S2f, g). In summary, a well-positioned inter-protomer SS bond (e.g., between the GP1 head and GP2 IFL) can stabilize the EBOV GP trimer, with no adverse effect on antigenicity.

Crystallographic analysis of redesigned EBOV GP $\Delta$ muc trimers. To understand how the HR2 stalk and $H R 1_{C}$ mutations affect EBOV GP, we determined crystal structures for the unliganded GP $\Delta$ muc-foldon with $\mathrm{WL}^{2}$ and $\mathrm{WL}^{2} \mathrm{P}^{2}$ at 2.3 and $3.2 \AA$, respectively (Fig. S3 and Fig. 3). Both proteins showed a threelobed, chalice-like trimer structure ${ }^{40,66} . \mathrm{WL}^{2} / \mathrm{WL}^{2} \mathrm{P}^{2}$ yielded $\mathrm{Ca}$ root-mean-square deviations (RMSDs) of $0.9 / 1.6 \AA$ (for $367 / 382$ residues) at the single protomer level and 1.25/1.23 $\AA$ (for 1103/ 1145 residues) at the trimer level, respectively, relative to wildtype GP (PDB ID: 5JQ3) ${ }^{66}$. $\mathrm{WL}^{2} \mathrm{P}^{2}$ yielded a more complete structure than $\mathrm{WL}^{2}$ at the glycan cap (R302-V310) and HR2 stalk (I627D637) (Fig. S3 and Fig. 3). In the $\mathrm{WL}^{2} \mathrm{P}^{2}$ structure, the glycan cap covers the RBS with glycan moieties visible for N238/N257/N268 in GP1 and N563 in GP2. In the WL ${ }^{2}$ structure, the glycan cap covers the RBS with glycan moieties visible for N238/N257 in GP1 and N563/N618 in GP2 (Fig. S3b). GP1 consists mainly of $\beta$ strands, which form a broad semicircular groove that clamps the $\alpha 3$ helix and $\beta 19-\beta 20$ strands in GP2 (Fig. 3a). The T577P mutation appeared to have minimal effect on conformation of the $\mathrm{HR} 1_{\mathrm{C}}$ bend, as indicated by a Ca RMSD of $0.2 \AA$ for this 8 -aa segment (Fig. 3b, left). In the $\mathrm{WL}^{2} \mathrm{P}^{2}$ structure, the backbone carbonyl (CO) groups of R574, A575, and T576 in one protomer formed moderate-to-strong hydrogen bonds with the guanidinium moiety of R164 in an adjacent protomer, whereas only one $\mathrm{CO}-\mathrm{NH}$ distance was within the $3.6 \AA$ cutoff in wildtype GP $\Delta \mathrm{muc}^{66}$. We previously hypothesized that a bulky, inwardfacing W615 at the top of the coiled-coil destabilizes the HR2 stalk, whereas the W615L mutation would improve its packing (Fig. 1d). Indeed, W615L exerted a positive effect on the stalk structure (Fig. 3b, bottom center and right). The $\mathrm{C} a-\mathrm{Ca} /$ $\mathrm{C} \beta-\mathrm{C} \beta$ distances between two W615s of adjacent protomers in 
a

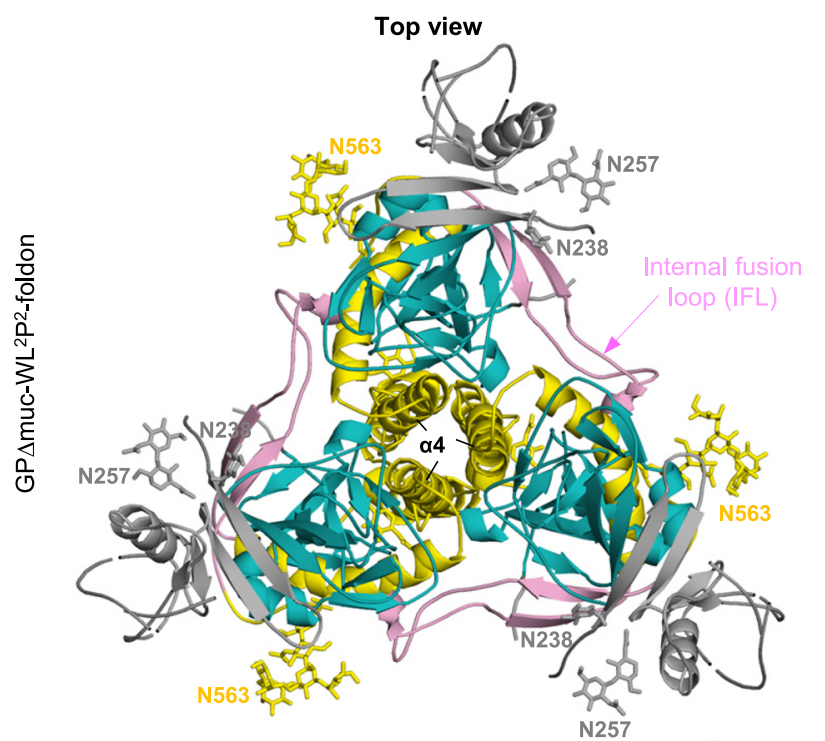

b

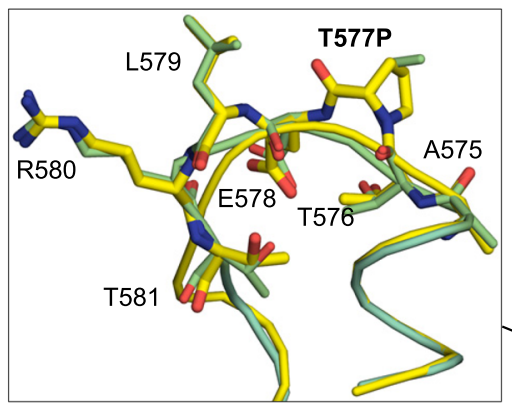

$H R 1_{C}$ bend $\left(W L^{2} P^{2}\right.$ vs. wildtype)

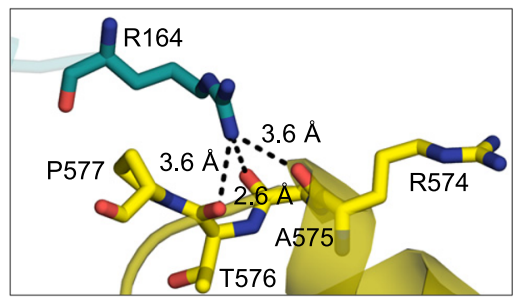

$W_{L}^{2} P^{2}: 3.2 \AA$ resolution

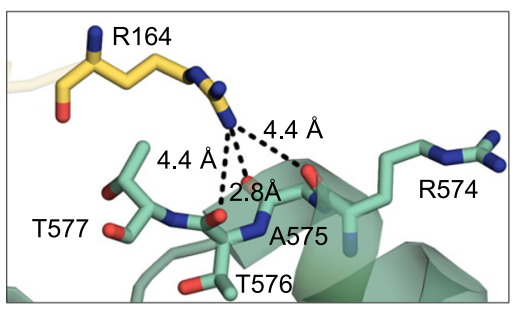

Wildtype (PDB ID: 5JQ3)

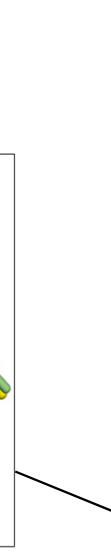

257

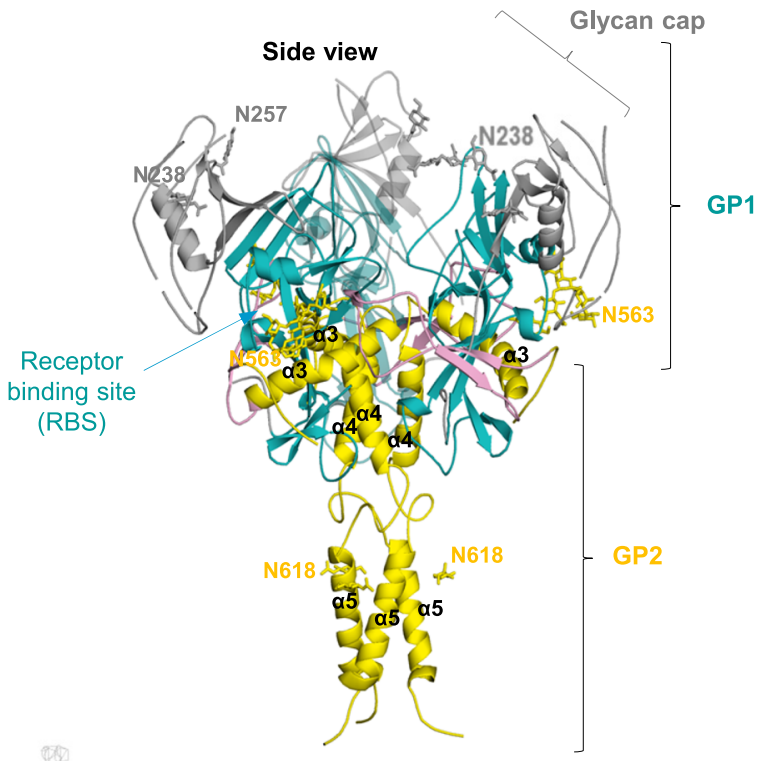

an cap 
Fig. 3 Structural characterization of EBOV GP with stalk and HR1 $\mathbf{C}_{\mathbf{C}}$ mutations. a The $3.2-\AA$-resolution crystal structure of EBOV GP $\Delta m u c-W L^{2} \mathrm{P}^{2}$-foldon in a ribbon representation (top view and side view). GP1 is shown in dodger blue, except for the glycan cap, which is in gray. GP2 is shown in yellow with the internal fusion loop (IFL) in pink. N-linked glycans at N238, N257, and N563 are shown as sticks. b Ribbon representation of a GP $\Delta$ muc-WL2P2 protomer in the center with inset images showing structural comparison for the HR1 $\mathrm{C}$ bend (left), W615-sorrounding HR2 region (bottom right), and $\mathrm{C}$ terminus of the HR2 stalk (right). For the HR1 1 bend, $W L^{2} \mathrm{P}^{2}$ is superimposed onto GP $\Delta$ muc (PDB ID: 5JQ3) (top) with three hydrogen bonds labeled for $W^{2} P^{2}$ (middle) and GP $\Delta$ muc (bottom). For the W615-surrounding HR2 region, WL2P2 is superimposed onto GPAmuc (PDB ID: 5JQ3) (top) with the coiled-coil structure shown for GPAmuc (left) and WL2P2 (right). $\mathrm{C} \alpha$ and $\mathrm{C} \beta$ distances for residue 615 around the threefold axis are labeled. For the HR2 $\mathrm{C}$ terminus, $\mathrm{WL}^{2} \mathrm{P}^{2}$ is superimposed onto GP structures of SUDV (top), EBOV (middle), and BDBV (bottom) with C $\alpha$ RMSDs calculated after fitting. The 2 Fo-Fc electron density map contoured at $1 \sigma$ is shown as a gray mesh for the $\mathrm{WL}^{2} \mathrm{P}^{2}$ protomer (center) and HR2 stalks (right).

partial MPER. The superposition of HR2 stalks, from R596 up to D637, yielded Ca RMSDs of $1.5,2.1$, and $1.9 \AA$ relative to EBOVMayinga (PDB ID: 5JQ3), Sudan ebolavirus (SUDV) (PDB ID: 3S88), and Bundibugyo ebolavirus (BDBV) (PDB ID: 6EA5) GPs, respectively (Fig. 3b, right), suggesting inherent structural variability in this region.

The $\mathrm{WL}^{2} \mathrm{P}^{2}$ structure was then compared to a recently reported Makona GP $\Delta$ muc structure (PDB ID: 6VKM, $3.5 \AA$ ) that contained the T577P/K588F mutation (Fig. S4). In total, 353 of 398 residues in the $\mathrm{WL}^{2} \mathrm{P}^{2}$ structure matched the double mutant with a Ca RMSD of $0.9 \AA$. A more complete cathepsin cleavage loop (between $\beta 13$ and $\beta 14$, residues 190-210) was observed in $\mathrm{WL}^{2} \mathrm{P}^{2}$ than in the double mutant, showing 10 more flanking residues, five on each side, of the loop that bridges over the IFL and interacts with IFL-directed NAbs such as mAb100 ${ }^{42}$. In addition, more electron density was observed for the $\beta 18$ loop of the glycan cap (residues 294-310) and the stalk in $\mathrm{WL}^{2} \mathrm{P}^{2}$ than in the double mutant (Fig. S4b, top right). For the $\mathrm{HR} 1_{\mathrm{C}}$ bend, $\mathrm{WL}^{2} \mathrm{P}^{2}$ showed a $\mathrm{Ca}$ RMSD of $0.3 \AA$ and more favorable hydrogen bonding patterns (Fig. S4b, bottom left). A Ca RMSD of $1.7 \AA$ was obtained for the IFL region between the two structures (Fig. S4b, bottom right). Lastly, the $\mathrm{WL}^{2} \mathrm{P}^{2}$ structure was docked into a panel of known GP/antibody complexes (Fig. $\mathrm{S} 5 \mathrm{a})$. Overall, $\mathrm{WL}^{2} \mathrm{P}^{2}$ preserved all critical GP-antibody interactions (Fig. S5b). The GP/mAb100 complex is of most interest, because mAb100 was successfully used in this study to purify GP trimers. Cryo-EM revealed additional density near the mAb100 light chain that likely corresponds to portions of the cathepsin cleavage loop ${ }^{42}$, but this density was not observed in a $6.7 \AA$ crystal structure of the same complex (PDB ID: 5FHC) ${ }^{42}$. In the $\mathrm{WL}^{2} \mathrm{P}^{2}$ structure, the flanking region on the $\beta 13$ side extended to H197 (Fig. S4), which would be in proximity to the mAb100 light chain in the $\mathrm{WL}^{2} \mathrm{P}^{2} / \mathrm{mAb} 100$ complex.

The crystal structures validated the stalk mutation $\mathrm{WL}^{2}$ and its combination with the $\mathrm{HR}_{\mathrm{C}}$ mutation, $\mathrm{WL}^{2} \mathrm{P}^{2}$, in addition to providing atomic details for regions that were absent in previously reported GP structures. The $\mathrm{WL}^{2} \mathrm{P}^{2}$ structure also provides an explanation for the higher trimer yield (i.e. the formation of more favorable inter-protomer hydrogen bonds), although the cause of the two-peak thermogram remains unclear. Notably, GP $\Delta$ muc-SS2 was not structurally characterized in this study, because its incompatibility with foldon posed a challenge to crystallization.

Display of EBOV GP $\Delta$ muc trimers on multilayered hyperstable nanoparticles. VLPs are intrinsically immunogenic due to their large size and dense antigen display ${ }^{88}$. Compared with small antigens, VLPs are more suitable for direct uptake by dendritic cells and clustering of B-cell receptors ${ }^{88}$. Although recombinant VLPs can protect against EBOV challenge ${ }^{60-62}$, they may not be optimal vaccine solutions because of abnormal filament structures (up to $14 \mu \mathrm{m}$ long) and manufacturing challenges ${ }^{63}$. Recently, self-assembling protein NPs were considered an alternative platform for developing VLP vaccines ${ }^{58,59}$. Previously, we displayed gp41-stabilized HIV-1 Env trimers on protein NPs of various sizes, which elicited robust $\mathrm{NAb}$ responses in mice and rabbits $^{55,64}$. We also reported protein NPs that present an optimized HCV E2 core, which induced cross-genotype NAb responses in mice ${ }^{65}$. In this study, we displayed rationally redesigned GP $\Delta$ muc trimers on 24- and 60-meric protein NPs for in vivo assessment.

To explore this possibility, we modeled the EBOV GP $\Delta$ muc trimer on FR, E2p, and I3-01, resulting in GP-presenting NPs with diameters of 34.6, 45.9, and $49.2 \mathrm{~nm}$, respectively (Fig. 4a). Superposition of GP $\Delta$ muc $C$ termini onto FR and E2p N termini yielded Ca RMSDs of 7.0 and $5.5 \AA$, suggesting that GP $\Delta$ muc can be fused to FR with a short $\mathrm{G}_{4} \mathrm{~S}$ linker and to E2p without a linker, respectively. However, the large spacing between the $\mathrm{N}$ termini of I3-01 subunits $(\sim 50.5 \AA)$ requires a long linker to connect with the $\mathrm{C}$ termini of a GP $\Delta$ muc trimer, which form a long, narrow stalk. Computational modeling suggested a 10-aa $\left(\mathrm{G}_{4} \mathrm{~S}\right)_{2}$ linker, which would result in a Ca RMSD of $0.8 \AA$. Here, we first displayed two GP $\Delta$ muc trimers, wildtype and $\mathrm{WL}^{2} \mathrm{P}^{2}$, on FR, E2p, and I3-01 with a 5-aa linker, no linker, and a 10-aa linker, respectively. $\mathrm{WL}^{2} \mathrm{P}^{2}$, instead of $\mathrm{WL}^{2}$, was selected for $\mathrm{NP}$ display for its high trimer propensity and atomic structure. All six GP-NP fusion constructs were transiently expressed in 100-ml ExpiCHO cells followed by mAb100 purification and SEC on a Superose 6 10/300 GL column (Fig. 4b). WL ${ }^{2} \mathrm{P}^{2}$ outperformed wildtype GP $\Delta$ muc with greater NP yield and purity. Based on molecular weight (m.w.), the SEC peaks at $\sim 15 \mathrm{ml}$ correspond to the unassembled GP-NP species, suggesting an inherent instability for wildtype E2p and I3-01. The mAb100-purified GP $\Delta$ muc$\mathrm{WL}^{2} \mathrm{P}^{2}$-presenting $\mathrm{NP}$ samples were further analyzed by negative-stain EM (Fig. 4c), showing NPs mixed with impurities.

Previously, we demonstrated the use of a pan-reactive T-cell epitope both as a linker and as a built-in T-cell help in an HIV-1 Env-I3-01 NP construct ${ }^{55}$, suggesting that additional structural and functional components can be incorporated into such large 60-meric NPs. Here, we sought to reengineer the E2p and I3-01 NPs by fusing a dimeric LD to the $C$ terminus of an NP subunit and then a T-helper epitope to the $\mathrm{C}$ terminus of an LD (Fig. 4d). We hypothesized that each LD dimer can stabilize a non-covalent NP-forming interface from inside, and the T-cell epitopes can form a hydrophobic core at the center of a fully assembled NP. To test this hypothesis, we manually inspected 815 homodimers in the PDB and selected nine LDs of 100 residues or less (Fig. S6a). Based on structural compatibility, LDs 1-7 were tested for E2p, and five LDs (4-5 and 7-9) were tested for I3-01, all displaying $\mathrm{GP} \Delta \mathrm{muc}-\mathrm{WL}^{2} \mathrm{P}^{2}$. Following transient expression in $100-\mathrm{ml}$ ExpiCHO cells and mAb100 purification, 12 LD-containing NP samples were characterized by SEC (Fig. 4e). Notably, LD4 and LD7 increased the NP peak $\left(\mathrm{UV}_{280}\right.$ value) by 5 - and 2.5 -fold for E2p and I3-01, respectively, with substantially improved NP purity. The further incorporation of a T-cell epitope, PADRE, did not alter E2p properties but negatively impacted I3-01 (Fig. 4f). An I3-01 variant, termed I3-01v9 (or 1VLW-v9 in the previous study ${ }^{55}$ ), was found to retain the NP yield and purity (Fig. 4f). 
a
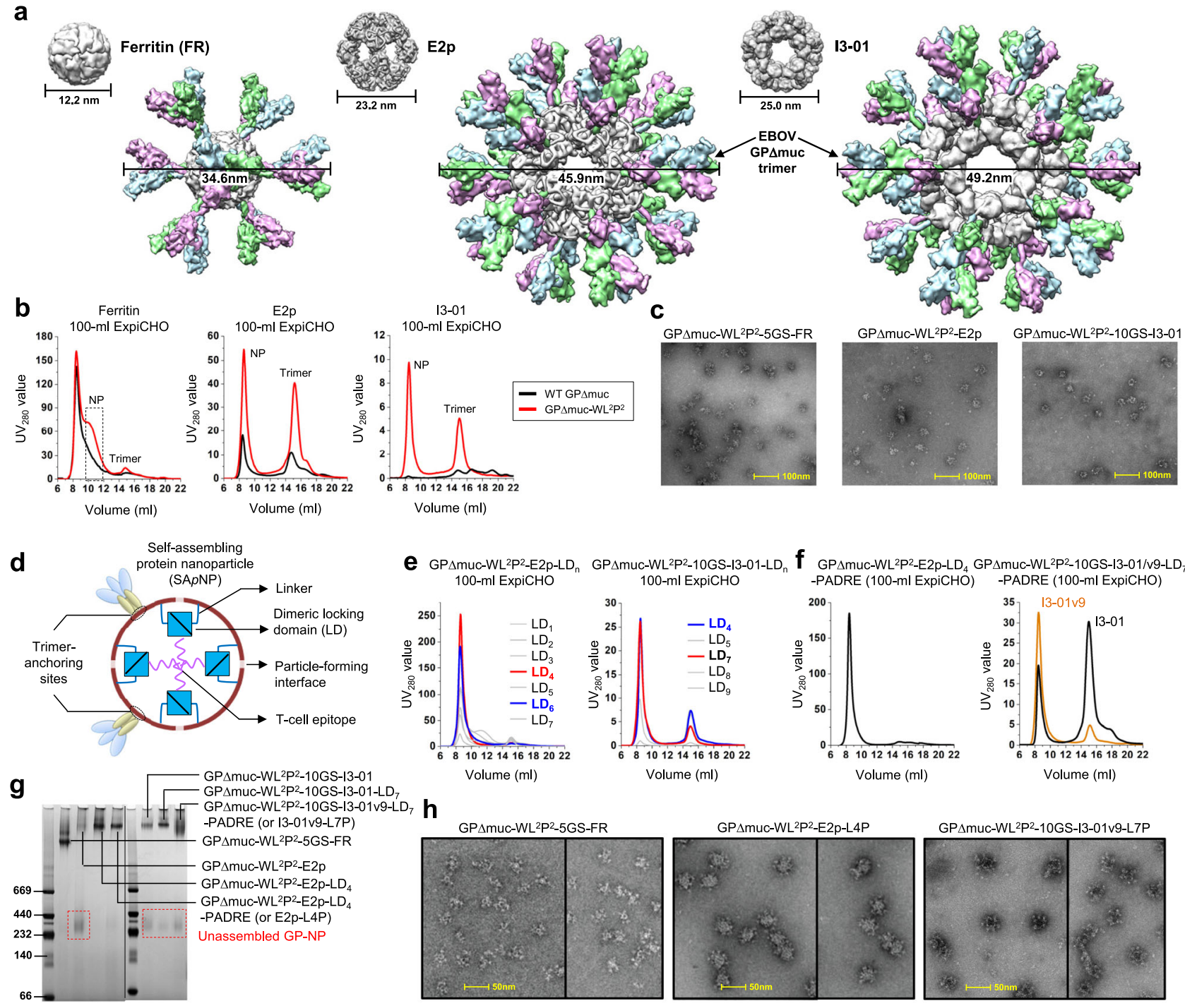

Volume $(\mathrm{ml})$
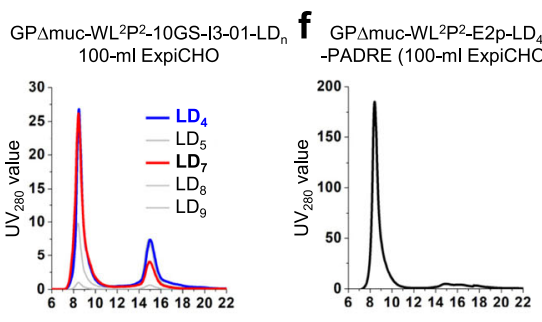

Volume ( $\mathrm{ml})$

Volume (ml)
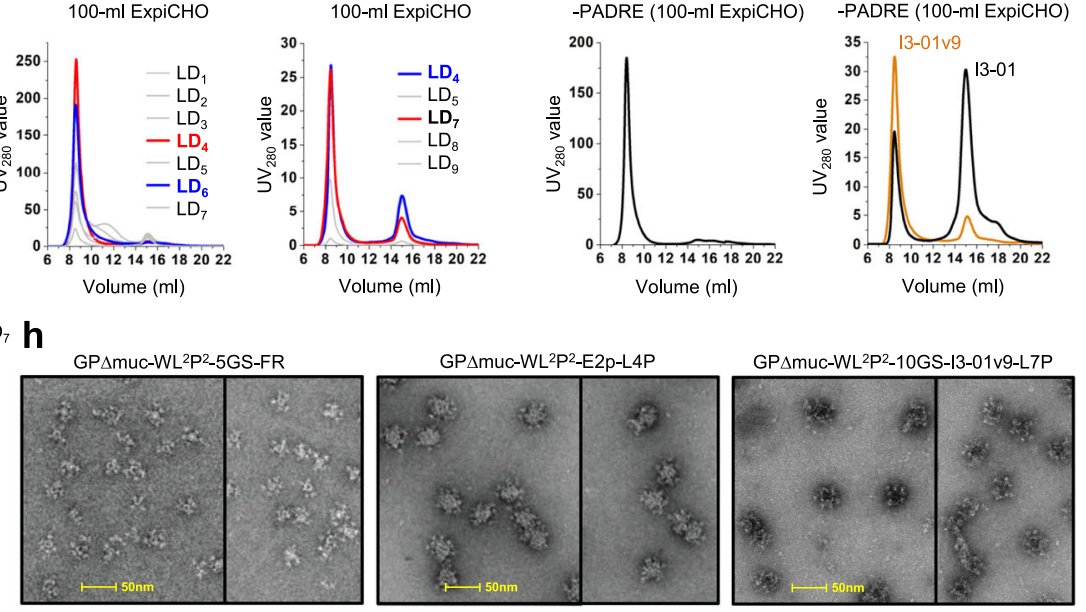

i

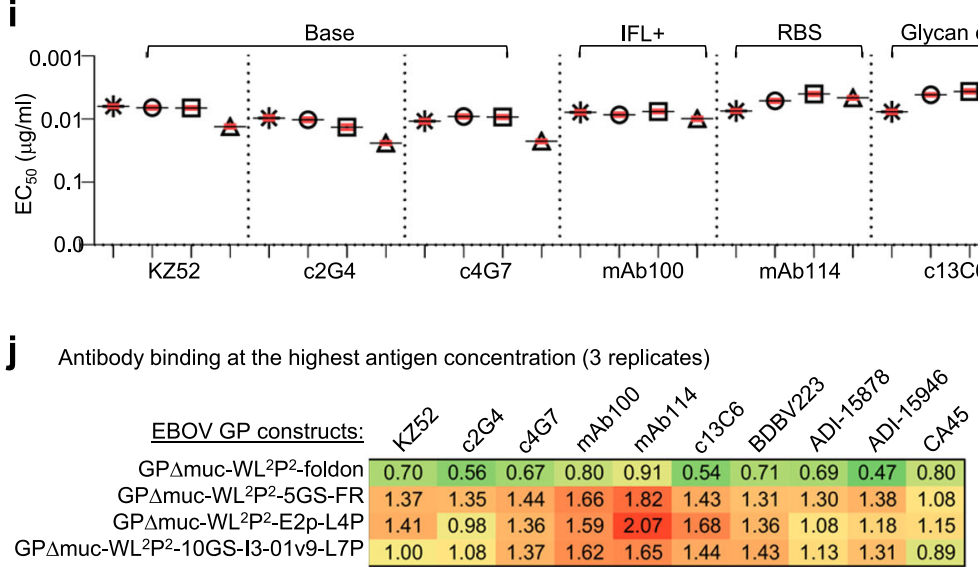

Seven GP-NP samples, with three variants for each 60-meric NP, were further analyzed by BN-PAGE (Fig. 4g). The FR and two E2p variants displayed a single high m.w. band corresponding to well-formed NPs, whereas the wildtype E2p and all three I3-01 samples showed additional low m.w. bands at $232-440 \mathrm{kD}$ on the BN gel, indicating unassembled GP-NP species. Lastly, the mAb100/SEC-purified FR, E2p-LD4-PADRE (E2p-L4P), and I3-
01v9-LD7-PADRE (I3-01v9-L7P) NPs that present the GP $\Delta$ muc$\mathrm{WL}^{2} \mathrm{P}^{2}$ trimer were analyzed by negative-stain EM (Fig. 4h). In addition to well-formed particles in all three samples, an array of GP $\Delta$ muc spikes could be clearly recognized on the surface of FR and E2p-L4P NPs.

Antigenicity of the three GP $\Delta$ muc- $\mathrm{WL}^{2} \mathrm{P}^{2}$-presenting NPs was assessed by ELISA using the same panel of 10 antibodies (Fig. $4 \mathrm{i}$ 
Fig. 4 Design and characterization of EBOV GPAMuc-presenting nanoparticles. a Surface models of nanoparticle (NP) carriers and GP $\Delta$ Muc-presenting NPs. The three NP carriers shown here are 24-meric ferritin (FR) and 60-meric E2p and I3-01. The NP size is indicated by diameter (in nm). $\mathbf{b}$ SEC profiles of wildtype GPAMuc (black) and WL2P2 (magenta)-presenting FR, E2p, and 13-01 NPs obtained from a Superose 6 10/300 GL column after mAb100 purification. The particle fraction is indicated by a dotted-line box for FR. c Negative-stain EM images of SEC-purified GP $\Delta$ Muc-WL2P2-presenting FR, E2p, and 13-01 NPs. $\mathbf{d}$ Schematic representation of multilayered NP design, in which a dimeric locking domain (LD) is fused to the C terminus of an NP subunit, and a helper T-cell epitope (PADRE) is fused to the C terminus of an LD. e SEC profiles of GPAMuc-WL2P2-presenting E2p NPs with LDs 1-7 and I3-02 NPs with five LDs (4-5 and 7-9) after mAb100 purification. $f$ SEC profiles of GPAMuc-WL2P2-presenting E2p NP with LD4 and PADRE, or E2p-L4P (left), and 13-01/v9 NP with LD7 and PADRE, or 13-01/v9-L7P (right). 13-01v9 is a variant of 13-01 with a redesigned NP-forming interface. $\mathbf{g}$ BN-PAGE of GPAMucWL2P2-presenting FR, E2p, and I3-01/v9 NPs, with LD and PADRE variants included for E2p and I3-01/v9. Low molecular weight (m.w.) bands are circled with red dotted lines. Black line indicates the gels on the left and right were generated from two separate experiments. $\mathbf{h}$ Negative-stain EM images of SECpurified FR, E2p-L4P, and I3-01v9-L7P NPs that present the GP $\Delta$ Muc-WL2 $\mathrm{P}^{2}$ trimer. Samples are shown as a composite of two panels, each representing a different micrograph. i EC 50 ( $\mu \mathrm{g} / \mathrm{ml})$ values of GP $\Delta$ Muc-WL2 $\mathrm{P}^{2}$-foldon and GP $\Delta$ Muc-WL2 $\mathrm{P}^{2}$-presenting NPs binding to 10 respective antibodies. Four panebolavirus NAbs are colored in red. Antibody binding was measured by ELISA in duplicates, with mean value and standard deviation (SD) shown as black and red lines, respectively. $\mathbf{j}$ Antigenic profiles of GP $\Delta M$ Muc-WL2 $\mathrm{P}^{2}$-foldon and GP $\Delta M$ Muc-WL2 $\mathrm{P}^{2}$-presenting NPs against 10 antibodies. Two BLI experiments were performed with three replicates tested for the highest antigen concentration. Sensorgrams were obtained from an Octet RED96 using an antigen titration series of six concentrations ( $400-12.5 \mathrm{nM}$ by twofold dilution for trimer, $25-0.78 \mathrm{nM}$ by twofold dilution for FR, and 10-0.31 nM for multilayered $\mathrm{E} 2 \mathrm{p}$ and $\mathrm{I3}-01 \mathrm{v} 9$ ) and quantitation (AHQ) biosensors. The average peak signals ( $\mathrm{nm}$ ) at the highest antigen concentration are listed in the matrix with the standard deviation (SD) shown in Fig. S6f. A higher color intensity indicates greater binding signal. Source data are provided as a Source Data file.

and Fig. S6b, c). Compared with the $\mathrm{WL}^{2} \mathrm{P}^{2}$ trimer, the three NPs exhibited an epitope-specific binding pattern. Overall, multivalent display improved antibody recognition of the RBS and glycan cap in GP1, but reduced binding for bNAbs that target the base and IFL at the GP1/GP2 interface (e.g., CA45) and the GP2 stalk (e.g., BDBV223). This finding raised concerns that some conserved bNAb epitopes on the NP-displayed GP trimers may not be as accessible as on the soluble GP trimers. Two BLI experiments, with a total of three replicates used for the highest antigen concentration, were performed to further characterize the effect of multivalent display on the antibody recognition of various GP epitopes (Fig. 4j and Fig. S6d-f). Using comparable GP molar concentrations, the three NPs showed higher binding signals than the soluble trimer, with the most notable differences for NAbs mAb114 and mAb100 and a non-NAb c13C6. Based on these results, the FR, E2p-L4P, and I3-01v9-L7P NPs that present the redesigned $\mathrm{GP} \Delta$ muc-WL ${ }^{2} \mathrm{P}^{2}$ trimer were selected for animal immunization.

Our results indicate that EBOV GP can be displayed on selfassembling NPs through gene fusion, which requires the optimization of both GP and NP. In addition to GP $\Delta$ muc, $\mathrm{GP}_{\mathrm{ECTO}}$ was also tested but found unsuitable for NP display, as confirmed by the EM analysis of a $\mathrm{GP}_{\mathrm{ECTO}^{-}}$10GS-FR construct (Fig. S6h). In this study, the multilayered NP design exploits the inner space of large, cage-like NPs to increase their stability and deliver additional $\mathrm{T}$-cell signals within a single-component system, representing a distinct strategy compared with the twocomponent NP design ${ }^{89,90}$.

Immunogenicity of EBOV GP trimers and NPs in BALB/c mice. Following the protocol in our previous $\mathrm{HIV}-1$ and $\mathrm{HCV}$ studies $^{55,65}$, we immunized BALB/c mice to assess the immunogenicity of six representative constructs, including three EBOV GP/GP $\Delta$ muc trimers and three NPs (Fig. 5a). A $\mathrm{GP}_{\text {ECTO-foldon }}$ trimer was included as a soluble version of the wildtype GP. Mice in the GP $\Delta$ muc-WL2 $\mathrm{P}^{2}-10 \mathrm{GS}-\mathrm{I3}-01 \mathrm{v} 9-\mathrm{L} 7 \mathrm{P}$ group were immunized with $20 \mu \mathrm{g}$ mAb100-purified protein instead of $50 \mu \mathrm{g}$ $\mathrm{mAb} 100 / \mathrm{SEC}$-purified protein due to the low yield of this NP. We first determined the GP-specific plasma antibody response in the ELISA using GP $\Delta$ muc-WL $\mathrm{L}^{2} \mathrm{P}^{2}-1 \mathrm{TD} 0$ as a probe, which utilized trimerization motif 1TD0 (PDB ID: 1TD0) (Fig. 5b and Fig. S7ac). The two GP $\Delta$ muc groups significantly $(P<0.0064)$ outperformed the $\mathrm{GP}_{\mathrm{ECTO}}$ group throughout immunization (Fig. 5b, top), suggesting that the MLD can shield GP from antibody recognition. However, little difference was found between the two
GP $\Delta$ muc groups, with $\mathrm{WL}^{2} \mathrm{P}^{2}$ showing a slightly higher average $\mathrm{EC}_{50}$ titer at $\mathrm{w} 2$ and $\mathrm{w} 5$ that was reversed at later time points. Compared with the $\mathrm{WL}^{2} \mathrm{P}^{2}$ trimer group, all $\mathrm{NP}$ groups showed lower $\mathrm{EC}_{50}$ titers except for the E2p-L4P NP group, which yielded a modest $P$ value of 0.0381 at w2 (Fig. 5b, bottom). In our recent HCV study, two NPs elicited higher antibody titers than the soluble E2 core at w2 $(P<0.0001)$ and w5 $(P \leq 0.0223)^{65}$. The stark difference between these two studies suggests that antibody titers in response to such NP vaccines may be influenced by antigen size, structure, and epitope distribution. NP display may occlude antibody access to the base and stalk epitopes, which are targets of many bNAbs ${ }^{9}$. This result may also be attributed to other factors, such as dosage, as noted in our recent study ${ }^{65}$. The NP carrier accounts for $21-33 \%$ of the total mass of an NP vaccine, and the same antigen dose $(50 \mu \mathrm{g})$ has been used for all groups except the I3-01v9-L7P NP group. Thus, mice in the NP groups would receive less GP antigen than mice in the trimeronly group.

Before analyzing the mouse plasma NAb response, we validated the pseudoparticle (pp) neutralization assay ${ }^{12}$ by testing 10 antibodies against two ebolavirus strains, EBOV-Makona and BDBV-Uganda, in $293 \mathrm{~T}$ and TZM-bl ${ }^{72}$ cells (Fig. 5c and Fig. S7d-f). mAb114 ${ }^{12}$ and early EBOV NAbs KZ52 ${ }^{76}, \mathrm{c} 2 \mathrm{G} 4$, and $c 47^{10}$ only neutralized EBOV-Makona, whereas $\mathrm{mAb} 100^{12}$ and four bNAbs, except BDBV2239 ${ }^{9}$, blocked both ebolavirus-pps. ADI-15946 was the most potent bNAb, as indicated by the half maximal inhibitory concentration $\left(\mathrm{IC}_{50}\right)$. Non-NAb c13C6, which is part of the ZMapp cocktail ${ }^{10}$ and binds the glycan cap ${ }^{45}$, enhanced ebolavirus-pp infection of both cell types. When tested against pseudoparticles bearing the murine leukemia virus (MLV) Env, MLV-pps, all antibodies were non-reactive, except for c13C6, which enhanced MLV-pp infection in 293T cells (Fig. $\mathrm{S7g})$. Overall, the enhancement observed for non-NAb c13C6 in the pseudovirus assays appeared to be consistent with $\mathrm{ADE}$ observed for human mAbs targeting the same epitope ${ }^{25}$.

We next performed neutralization assays using purified mouse immunoglobulin $\mathrm{G}$ (IgG) from the last time point, w11 (Fig. 5d and Fig. S7h, i). Two distinct types of antibody response were observed: NAbs and non-NAbs that enhanced ebolavirus-pp infection. Among the three GP trimers, GP ECTO elicited a moderate $\mathrm{NAb}$ response with signs of enhancement noted for three mice, whereas an increase in both types of antibody response was observed for GP $\Delta$ muc, suggesting that the removal of MLD exposes both NAb epitopes and the glycan cap, which is a main target for ADE-causing human $m A b s^{25}$. The stalk/HR $1_{C}$ 
a

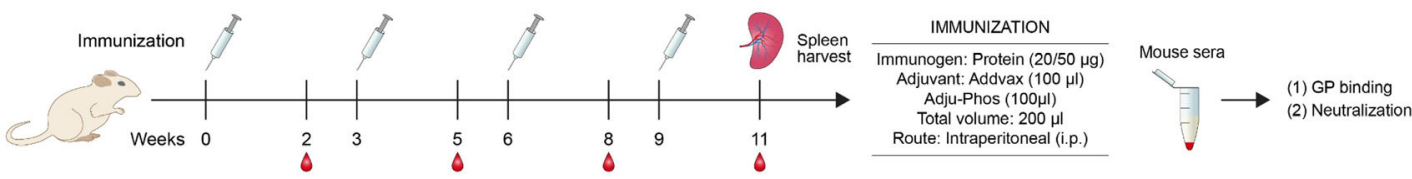

b Mouse antibody response
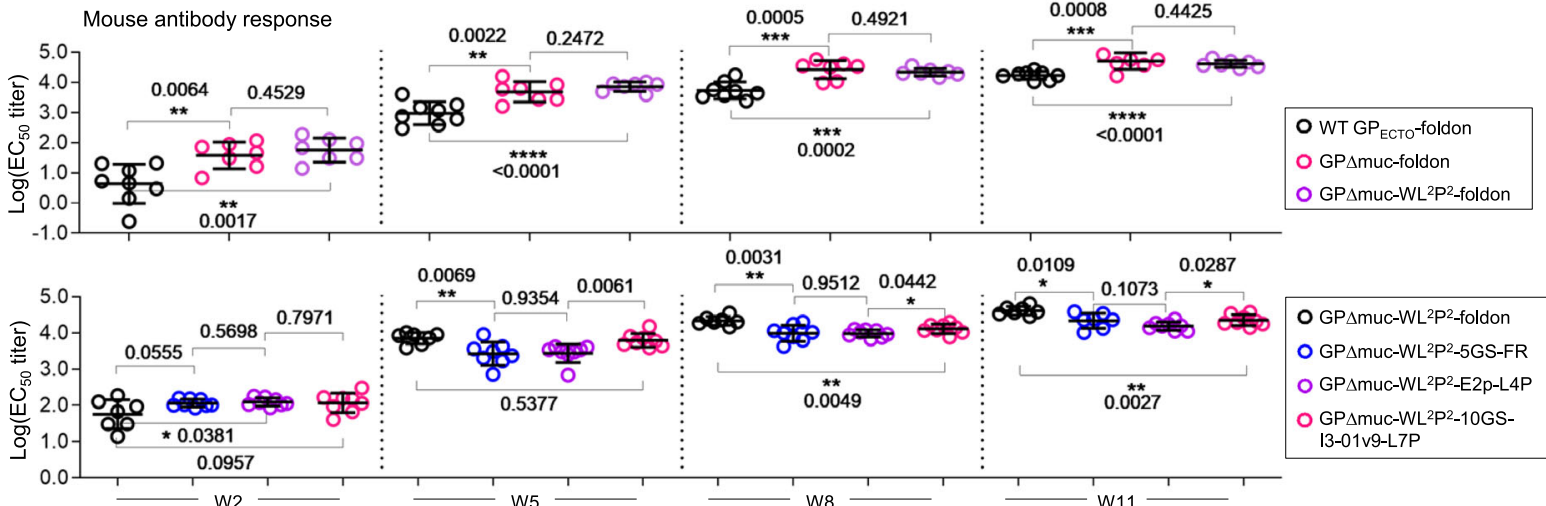

C

\begin{tabular}{|c|c|c|c|c|c|c|c|c|c|c|}
\hline & \multicolumn{5}{|c|}{ NAbs } & \multicolumn{4}{|c|}{ Cross-reactive NAbs } & \multirow{2}{*}{$\begin{array}{c}\text { Non-NAb } \\
\mathrm{c} 13 \mathrm{C} 6 \\
\end{array}$} \\
\hline & KZ52 & $c 2 \mathrm{G} 4$ & $c 4 G 7$ & mAb100 & mAb114 & BDBV223 & ADI-1587 & ADI-15946 & CA45 & \\
\hline EBOV-Makona & 5.6 & 4.2 & 3.6 & 5.9 & 4.2 & $>10$ & 2.9 & 2.2 & $9.9 \mathrm{E}-01$ & $>10^{*}$ \\
\hline BDBV-Uganda & $>10$ & $>10$ & $>10$ & 1.8E-01 & $>10$ & $1.2 \mathrm{E}-01$ & $3.4 \mathrm{E}-02$ & 2.7E- -02 & $7.8 \mathrm{E}-02$ & $>10^{*}$ \\
\hline
\end{tabular}

d

GP trimer and nanoparticle-induced mixed NAb/non-NAb responses in mice at week $11^{\mathrm{a}}$

\begin{tabular}{|c|c|c|c|c|c|c|c|c|c|c|c|c|c|c|c|c|}
\hline \multicolumn{9}{|c|}{\begin{tabular}{l|l|l|l|l|} 
& \multicolumn{3}{c}{ EBOV-Makona } \\
\cline { 2 - 4 }
\end{tabular}} & \multicolumn{8}{|c|}{ BDBV-Uganda } \\
\hline & $\mathrm{M} 1$ & $\mathrm{M} 2$ & M3 & M4 & M5 & M6 & M7 & M8 & M1 & M2 & M3 & M4 & M5 & M6 & M7 & M8 \\
\hline GP $\triangle$ muc-foldon & $>10^{3 *}$ & $>10^{3 *}$ & $>10^{3 *}$ & $>10^{3 *}$ & 102 & $>10^{3}$ & 282 & 110 & 252 & $788^{*}$ & $>10^{3 *}$ & 23 & 27 & 380 & 795 & 115 \\
\hline GP $\Delta$ muc-WL22 P $^{2}-5 G S-F R$ & 408 & 136 & 250 & $>10^{3 *}$ & 609 & - & 82 & 105 & 582 & 130 & $>10^{3 *}$ & $>10^{3 *}$ & $>10^{3 *}$ & - & 95 & 395 \\
\hline GP $\triangle m u c-W L^{2} P^{2}-E 2 p-L 4 P$ & 76 & 131 & 94 & 140 & 141 & 100 & 85 & 185 & $>10^{3}$ & 31 & 23 & 486 & 31 & 16 & 346 & 65 \\
\hline GP $\Delta$ muc-WL2P2-10GS-13-01-L7P b & $>10^{3 *}$ & $>10^{3 *}$ & $>10^{3 *}$ & $>10^{3 *}$ & $>10^{3}$ * & $>10^{3 *}$ & $>10^{3 *}$ & $>10^{3}$ & $>10^{3 \star}$ & 318 & $>10^{3 *}$ & 782 & $>10^{3 *}$ & $>10^{3} *$ & $>10^{3 *}$ & $>10^{3 *}$ \\
\hline
\end{tabular}

e

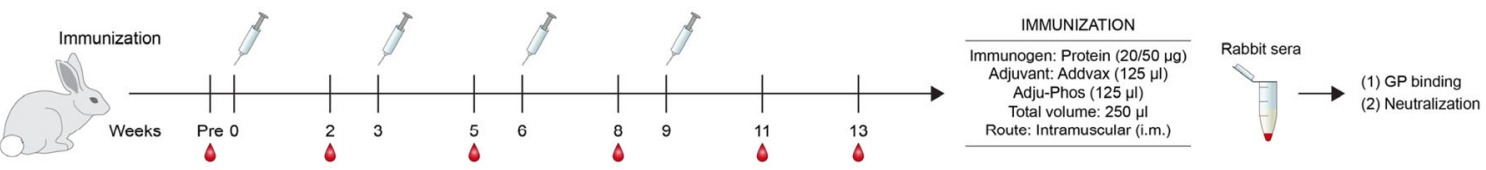

$\mathbf{f}$

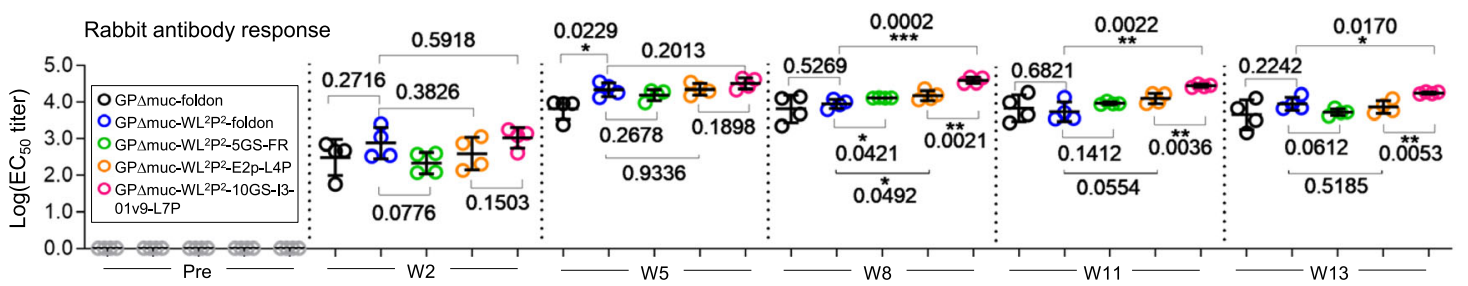

\section{g}

\begin{tabular}{|c|c|c|c|c|c|c|c|c|}
\hline & \multicolumn{4}{|c|}{ EBOV-Makona } & \multicolumn{4}{|c|}{ BDBV-Uganda } \\
\hline & R1 & $\mathrm{R} 2$ & R3 & $\mathrm{R} 4$ & R1 & $\mathrm{R} 2$ & $\mathrm{R} 3$ & R4 \\
\hline GP $\triangle$ muc-foldon & 332 & $>1000$ & 278 & $>1000$ & 12 & 18 & 19 & 58 \\
\hline GP $\triangle$ muc-WL2P2-foldon & 309 & 399 & $>1000$ & 541 & 9 & 14 & 27 & 42 \\
\hline GPAmuc-WL2²2-5GS-FR & 507 & 299 & 349 & 475 & 43 & 17 & 16 & 26 \\
\hline GPAmuc-WL2P2-E2p-L4P & 454 & 325 & $>1000$ & 432 & 24 & 11 & 41 & 14 \\
\hline GP $\triangle$ muc-WL2P2-10GS-13-01-L7P b & 216 & 141 & 257 & 231 & 18 & 10 & 9 & 10 \\
\hline
\end{tabular}

mutation $\mathrm{WL}^{2} \mathrm{P}^{2}$ appeared to have largely reversed the enhancement caused by MLD removal. Among the three NPs, E2p-L4P elicited primarily NAb responses that blocked both ebolaviruspps, whereas enhanced pseudoviral infection was observed for one mouse in the FR group and for all mice in the I3-01v9-L7P group. Because only non-NAb c13C6 and not any of the (b)NAbs reacted with MLV-pps (Fig. S7g), here we utilized the MLV-pp assay to gauge glycan cap-directed non-NAb responses induced by different vaccine constructs (Fig. S7j). Indeed, the patterns of enhanced MLV-pp infection correlated nicely with the patterns of enhanced ebolavirus-pp infection (Fig. S7h-j). In the MLV-pp assay, E2p-L4P NP induced a minimum enhancement-causing 
Fig. 5 Immunogenicity of EBOV GPAMuc trimers and nanoparticles in mice. a Schematic representation of the mouse immunization protocol. $\mathbf{b}$ Longitudinal analysis of GP-specific antibody titers in immunized mouse plasma $(n=8)$ at w2, w5, w8, and w11. Top panel: $\mathrm{EC}_{50}$ titers (fold of dilution) calculated from ELISA binding of mouse plasma from three GP trimer groups to the coating antigen, GP $\Delta$ Muc-WL2P2. Bottom panel: $E C_{50}$ titers calculated from ELISA binding of mouse plasma from three NP groups to the coating antigen, GP $\Delta$ Muc-WL2 $\mathrm{P}^{2}$, with the GP $\Delta$ Muc-WL2 $\mathrm{P}^{2}$-foldon group included for comparison. Mean values and standard deviation (SD) are shown as black lines. $P$ values were determined by an unpaired two-tailed $t$ test in GraphPad Prism 8.4.3 and are labeled on the plots. The asterisk symbol $\left({ }^{\star}\right)$ indicates the level of statistical significance: ${ }^{\star} P<0.05$; ${ }^{\star \star} P<0.01 ;{ }^{\star \star \star} P<0.001$; ${ }^{\star \star \star \star} P<$ 0.0001. c Ebolavirus-pp (EBOV-Makona and BDBV-Uganda) neutralization by 10 antibodies in 293T cells. The neutralization was performed in duplicates. The half maximal inhibitory concentration $\left(\mathrm{IC}_{50}\right)$ values are listed with the enhanced pseudovirus infection $(<-30 \%)$ indicated by an asterisk $\left({ }^{*}\right)$. $\mathbf{d}$ Ebolavirus-pp (EBOV-Makona and BDBV-Uganda) neutralization by purified mouse IgGs from six vaccine groups in 293T cells. The neutralization was performed without duplicates due to limited sample availability. e Schematic representation of the rabbit immunization protocol. $\mathbf{f}$ Longitudinal analysis of GP-specific antibody titers in immunized rabbit sera $(n=4)$ at weeks $0,2,5,8,11$, and 13. Mean values and standard deviation (SD) are shown as black lines. $\mathbf{g}$ Ebolavirus-pp (EBOV-Makona and BDBV-Uganda) neutralization by purified rabbit IgGs from five vaccine groups in 293T cells. The neutralization was performed in duplicates. In $\mathbf{d}, \mathbf{g}$, due to the presence of enhancement-causing non-NAbs in the purified IGs, approximate $I_{50}$ values derived from the fitting of \% neutralization data are listed for comparison, with the enhanced pseudovirus infection $(<-30 \%)$ indicated by an asterisk $\left({ }^{\star}\right)$. Source data are provided as a Source Data file.

non-NAb response at a similar level to $\mathrm{GP}_{\mathrm{ECTO}}$, in which MLD shields the glycan cap and other GP epitopes from the humoral response.

Our mouse study thus revealed some salient features of the GPspecific antibody response in the context of various GP forms and NP carriers. The c13C6-like non-NAbs that bind the glycan cap and cross-react with small secreted $\mathrm{GP}^{45}$ need to be minimized in vaccine design. The high level of enhancement-causing non-NAb responses observed for GP $\Delta$ muc and I3-01v9-L7P may be explained by less trimeric GP and unassembled GP-NP species, respectively. Nonetheless, a multilayered E2p NP displaying 20 $\mathrm{GP} \Delta$ muc trimers elicited a robust bNAb response in mice.

Immunogenicity of EBOV GP trimers and NPs in rabbits. Following a similar protocol, we assessed two GP $\Delta$ muc trimers, wildtype and $\mathrm{WL}^{2} \mathrm{P}^{2}$, and three NPs presenting the $\mathrm{WL}^{2} \mathrm{P}^{2}$ trimer in rabbits (Fig. 5e). Rabbit sera collected at six timepoints during immunization were analyzed by ELISA using the same trimer probe (Fig. $5 \mathrm{f}$ and Fig. S8a, b). Notably, rabbits in the I3-01v9L7P NP group were immunized with $20 \mu \mathrm{g}$ of mAb100/SECpurified material to reduce the enhancement-causing non-NAbs. Between the two trimer groups, $\mathrm{WL}^{2} \mathrm{P}^{2}$ showed higher average $\mathrm{EC}_{50}$ titers for all time points except w11, with a modest $P$ value of 0.0229 at w5. Among the three NP groups, the I3-01v9 and FR groups yielded the highest and lowest $\mathrm{EC}_{50}$ titers, respectively, throughout immunization. A significant difference was found between the I3-01v9-L7P and E2p-L4P groups at w8, w11, and w13, with $P$ values in the range of $0.0021-0.0053$. Compared with the GP $\Delta$ muc-WL ${ }^{2} \mathrm{P}^{2}$ group, the I3-01v9-L7P NP group showed higher $\mathrm{EC}_{50}$ titers at all six time points, with significant $P$ values at w8, w11, and w13. In contrast, the FR and E2p-L4P groups yielded lower $\mathrm{EC}_{50}$ titers than the GP $\Delta$ muc-WL $\mathrm{W}^{2} \mathrm{P}^{2}$ group at $\mathrm{w} 2$ and $\mathrm{w} 5$, but this pattern was reversed at $\mathrm{w} 8$ and w11 with modest $P$ values at w8. However, these two NP groups ended with lower $\mathrm{EC}_{50}$ titers than the trimer group at the last time point, w13.

We then performed ebolavirus-pp and MLV-pp assays using purified rabbit IgG from w11 (Fig. $5 g$ and Fig. S8c). At this time point, all vaccine groups showed NAb responses with no sign of enhanced pseudovirus infection, in contrast to the pattern of mixed antibody responses in mice (Fig. 5d). Notably, the I3-01v9L7P NP group yielded higher average $\mathrm{IC}_{50}$ titers than the other groups, 211.3 and $11.72 \mu \mathrm{g} / \mathrm{ml}$ for EBOV-Makona and BDBVUganda, respectively, supporting the notion that unassembled GP-NP species and not the I3-01v9 NP carrier were responsible for eliciting enhancement-causing non-NAbs in mice. All vaccine groups showed no sign of enhanced MLV-pp infection at w11 (Fig. S8c). Therefore, enhancement-causing non-NAbs appeared to be absent in rabbit sera toward the end of immunization. We next analyzed rabbit IgG from earlier time points at day 0 (Pre), w2, w5, and w8 (Fig. S8d-g), which revealed a unique temporal pattern of an increasing NAb response tailing a transient enhancement-causing non-NAb response. Specifically, enhanced MLV-pp infection was observed for the two trimer groups, FR group, and two multilayered NP groups at w2, w5, and w8, which then disappeared at w5, w8, and w11, respectively. Our longitudinal analysis suggests that vaccine-induced enhancement-causing nonNAbs may shift epitopes and gain neutralizing activity through gene conversion, a mechanism employed by the rabbit immune system to rapidly develop functional antibodies ${ }^{91}$.

B-cell response profiles associated with EBOV GP trimers and NPs. Previously, we combined antigen-specific B-cell sorting and NGS to obtain a quantitative readout of the B-cell response induced by an HCV E2 core and its E2p NP65. A more diverse usage of heavy-chain variable genes $\left(\mathrm{V}_{\mathrm{H}}\right)$, a higher degree of $\mathrm{V}_{\mathrm{H}}$ mutations, and a broader range of heavy-chain complementarity determining region 3 (HCDR3) length were observed for $\mathrm{E} 2 \mathrm{p}^{65}$. In this study, we applied the same strategy to obtain GP-specific B-cell profiles (Fig. 6a). Using an Avi-tagged GP $\Delta$ muc-WL ${ }^{2} \mathrm{P}^{2}$ 1TD0 probe (Fig. S9a), we sorted GP-specific splenic B cells from 25 mice (Fig. S9b), which were sequenced on Ion GeneStudio S5. The NGS data were analyzed using a mouse Antibodyomics pipeline $^{92}$ (Fig. S9c), with quantitative B-cell profiles derived for different vaccine groups (Fig. $6 \mathrm{~b}$ and Fig. S9d-f). We mainly focused on the GP $\Delta$ muc-WL ${ }^{2} \mathrm{P}^{2}$-foldon group and multilayered E2p group to compare B-cell responses to GP $\Delta$ muc in the trimeric versus NP forms (Fig. 6b). In terms of germline gene usage, similar patterns were observed for $\mathrm{V}_{\mathrm{H}}$ and $\mathrm{V}_{\mathrm{K}}$ genes (Fig. 6b, panels 1 and 2). The redesigned GP $\Delta$ muc trimer activated more $\mathrm{V}_{\mathrm{H}} / \mathrm{V}_{\mathrm{L}}$ genes $(9.4 / 9.4)$ than its NP form (6/7), with $P$ values of 0.0163 and 0.0076 for $V_{H}$ and $V_{L}$ genes, respectively. In contrast, the E2p NP decorated with $60 \mathrm{HCV} \mathrm{E} 2$ cores activated more $\mathrm{V}_{\mathrm{H}}$ but not $\mathrm{V}_{\mathrm{L}}$ genes than the $\mathrm{E} 2$ core $^{65}$. In terms of somatic hypermutation (SHM), no significant difference was found between the two groups (Fig. 6b, panel 3). However, we observed a visible shift in the SHM distribution for the E2p-L4P NP group, which showed higher germline $\mathrm{V}_{\mathrm{H}} / \mathrm{V}_{\mathrm{K}}$ divergence (on average $6.4 \% / 2.9 \%$ ) than the trimer group (on average 5.3\%/2.6\%). In the HCDR3 analysis, both average loop length and the RMS fluctuation (RMSF) of loop length were calculated (Fig. 6b, panel 4). Unlike in the HCV study, in which the RMSF of HCDR3 length yielded a $P<0.0001$ between the E2 core and E2p NP groups ${ }^{65}$, no significant difference was found between the EBOV GP $\triangle$ muc and E2p-L4P NP groups. Overall, EBOV and HCV NPs exhibited distinct patterns of the B-cell response with respect to their individual antigens. Notably, there were no apparent correlations 
a
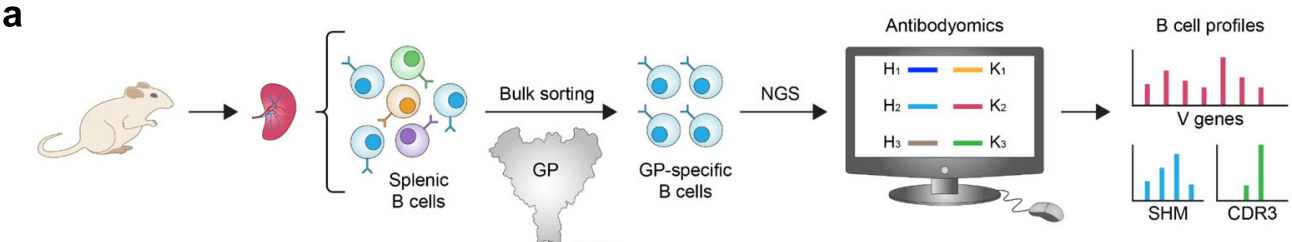

b
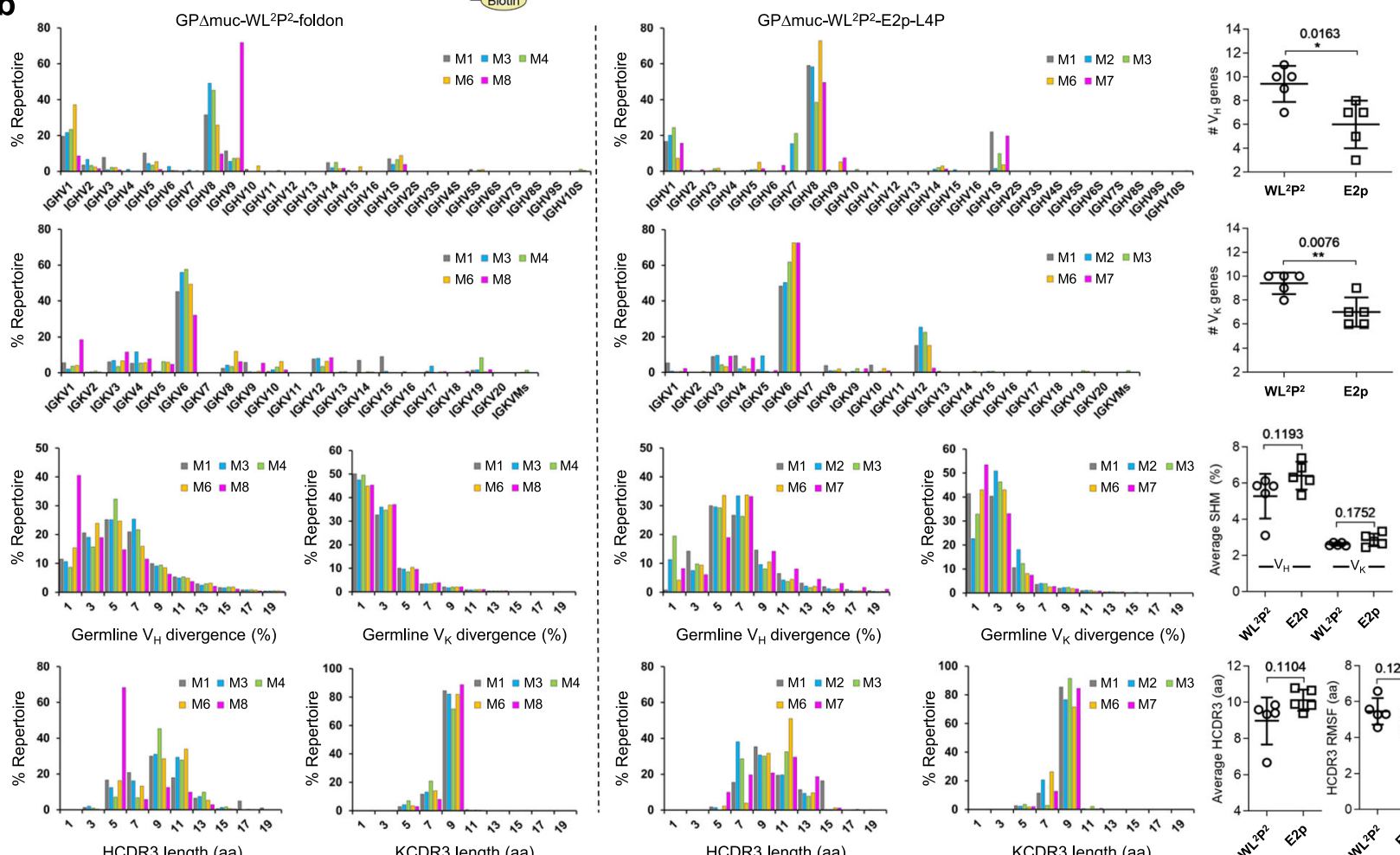

Fig. 6 Quantitative assessment of GP-specific B-cell response. a Schematic representation of the strategy to analyze the GP-specific murine B-cell response that combines the antigen-specific bulk sorting of splenic B cells with next-generation sequencing (NGS) and antibodyomics analysis. b B-cell profiles for the GPAMuc-WL22 2-foldon (left) and GP $\Delta M u c-W L^{2} P^{2}-E 2 p-L 4 P$ groups (right) and statistical comparison of key parameters derived from the profile analysis (far right). For each vaccine group $(n=8)$, five mice were selected for NGS analysis. Panel 1/2: Distribution of germline $V_{H} / V_{K}$ genes and statistical comparison of the number of activated $V_{H} / V_{K}$ genes ( $\geq 1 \%$ of the total population). Panel 3: distribution of $V_{H} / V_{K}$ somatic hypermutation (SHM) with percent (\%) mutation calculated at the nucleotide level, and statistical comparison of the average $\mathrm{V}_{\mathrm{H}} / \mathrm{V}_{\mathrm{K}} \mathrm{SHM}$ rate. Panel 4: distribution of $\mathrm{H} / \mathrm{KCDR} 3$ loop length and statistical comparison of two parameters, HCDR3 loop length and root-mean-square fluctuation (RMSF) of HCDR3 loop length. The RMSF value is used as an indicator of how much HCDR3 loop length varies within the EBOV GP-specific antibodies from each animal. In statistical comparison (far right), mean value and standard deviation (SD) are shown as black lines. $P$ values were determined by an unpaired two-tailed $t$ test in GraphPad Prism 8.4.3 and are labeled on the plots. The asterisk symbol $\left.{ }^{\star}\right)$ indicates the level of statistical significance: ${ }^{\star} P<0.05 ;{ }^{\star \star} P<0.01$; ${ }^{\star \star \star} P<0.001$. Source data are provided as a Source Data file.

between B-cell profiles and vaccine-induced $\mathrm{NAb}$ and enhancement-causing non-NAb responses. Our results thus suggest that antigen size, structure, glycosylation, and epitope distribution, other than the NP carrier, may contribute critically to the NP vaccine-induced B-cell response.

\section{Discussion}

With a mortality rate of up to $90 \%$, EBOV has caused significant humanitarian crises, calling for action across many sectors to develop effective interventions ${ }^{3}$. ZMapp ${ }^{10,11}$ established the use of NAbs as a treatment for patients with Ebola virus disease and propelled a community-wide campaign to identify NAbs and bNAbs $8,9,30,48$. Several vaccine candidates have been tested in human trials ${ }^{63,93}$. Of these, rVSV-ZEBOV demonstrated an efficacy of $100 \%$ in an open-label, cluster-randomized ring trial ${ }^{16}$ and was recently approved for human use. In a Phase 2 placebocontrolled trial of two vectored vaccines (including rVSV-
ZEBOV), the antibody titer remained similar between the vaccine and placebo groups at 1 week post-vaccination and peaked after 1 month ${ }^{15}$. A recent analysis of human B-cell responses to EBOV infection revealed hurdles in the GP-specific NAb response ${ }^{28,29}$. NAbs from vaccinated humans showed low levels of $\mathrm{SHM}^{94}$, suggesting a suboptimal B-cell response elicited by an otherwise effective vectored vaccine ${ }^{95}$. The immune correlates of vaccine protection may not be universal and are likely determined by vaccine platforms, in addition to other factors ${ }^{96}$. As most EBOV vaccines are based on viral vectors ${ }^{63,93}$, protein subunit vaccines remain a promising approach to combat this deadly virus.

Here, we approached EBOV vaccine design with an antibodybased strategy focusing on GP optimization and multivalent display. We applied a similar strategy to develop HIV-1 and HCV vaccine candidates for in vitro and in vivo characterization $55,56,64,65$, which provided a context for interpreting the findings for EBOV. Previously, we identified an $\mathrm{HR} 1_{\mathrm{N}}$ bend as the cause of HIV-1 Env metastability ${ }^{55,56}$ and optimized an HCV E2 core ${ }^{65}$. EBOV GP 
belongs to the class-I fusion protein family ${ }^{53,54}$ and is inherently metastable, as is HIV-1 Env. In this study, we probed the cause of EBOV GP metastability by testing various designs that target the $\mathrm{HR} 2$ stalk, $\mathrm{HR} 1_{\mathrm{C}}$ bend, and GP/GP interface (via inter-protomer disulfide bonds). The detailed characterization revealed a stabilizing effect of the W615L mutation and stalk extension, in addition to the unexpected sensitivity of $\mathrm{HR} 1_{\mathrm{C}}$ (equivalent to $\mathrm{HIV}-1 \mathrm{HR} 1_{\mathrm{N}}$ ) to specific proline mutations, which increased the trimer yield but caused complex unfolding behaviors in DSC. Because this pattern was not reported for EBOV-Makona GP $\Delta$ muc that contained the T577P mutation ${ }^{84}$, GP metastability may thus be a strain-specific feature and warrant further investigation. Multivalent NP display proved to be challenging for EBOV GP because of its tendency toward dissociation. Although two-component $\mathrm{NPs}^{89,90}$ and the SpyTag/SpyCatcher system ${ }^{97,98}$ have been used to develop VLP-type vaccines, their inherent complexity in production, assembly, and purification, structural instability in vivo, and off-target response may dampen their potential as human vaccines. Here, we designed single-component, multilayered, and self-assembling protein NPs based on E2p and I3-01v9. Encoded within a single plasmid, such $\mathrm{NP}$ vaccines can be readily produced in good manufacturing practice (GMP)-compatible CHO cells followed by IAC and SEC purification, providing a simple and robust manufacturing process. Our immunogenicity studies in mice and rabbits revealed some salient features of GP that need to be addressed in EBOV vaccine development, regardless of the delivery platform. The choice of $\mathrm{GP}_{\text {ЕCTO }}$ or GP $\Delta$ muc as a vaccine antigen may lead to antibody responses that target different GP epitopes. Antibody access to GP epitopes at the IFL, base, and HR2 stalk may differ in the trimeric and NP forms. In animal immunization, we observed an ADE-like non-NAb response, which may be associated with non-trimeric GP and unassembled GP-NP species. Antibody isolation, structural epitope mapping, and live EBOV neutralization assays may be required to determine the biological relevance of these findings. Furthermore, EBOV challenge in rodents may help determine protective NAb titers elicited by GP-presenting NPs with respect to recombinant $V_{L P s}{ }^{60-62}$. Nonetheless, the E2p-L4P NP elicited a minimum non$\mathrm{NAb}$ response in mice and the highly purified I3-01v9-L7P NP induced the strongest $\mathrm{NAb}$ response in rabbits, providing two promising constructs for further optimization and in vivo evaluation.

Having assessed various GP trimer and NP constructs, future investigation may be directed toward assessing other GP $\Delta$ muc designs, such as GP $\Delta$ muc-WL ${ }^{2}$ and GP $\Delta$ muc-SS2, as well as their $\mathrm{NPs}$, to further improve NAb responses and reduce glycan capdirected non- $\mathrm{NAb}$ responses. The structural characterization of NAbs and non-NAbs isolated from immunized animals will provide critical insights into epitope recognition and guide future vaccine design. The strategy described in this study may find applications in vaccine development for other filoviruses.

\footnotetext{
Methods

Design, expression, and purification of EBOV GPAmuc and GPAmucpresenting NPs. The glycoprotein sequence of Zaire EBOV (Mayinga-76 strain) with a T42A substitution was used to design all GP constructs in this study (UniProt ID: Q05320), with the primers summarized in Table S1. Logo analysis of EBOV and MARV GP sequences was performed using WebLoGo v2.8 software to facilitate the design of the W615L mutation. Structural modeling was performed using the UCSF Chimera v1.13 software to facilitate the design of HR $1_{C}$-proline and inter-protomer disulfide bond mutations. Wildtype and redesigned GP $\Delta$ muc constructs were transiently expressed in HEK293F cells (Thermo Fisher) for biochemical, biophysical, and antigenic analyses. Briefly, HEK293F cells were thawed and incubated with FreeStyle $\mathrm{T}^{\mathrm{TM}} 293$ Expression Medium (Life Technologies, CA) in a shaker incubator at $37^{\circ} \mathrm{C}$ at $135 \mathrm{rpm}$ with $8 \% \mathrm{CO}_{2}$. When the cells reached a density of $2.0 \times 10^{6} / \mathrm{ml}$, expression medium was added to reduce cell density to $1.0 \times 10^{6} \mathrm{ml}^{-1}$ for transfection with polyethyleneimine (PEI) (Polysciences, Inc). Next, $900 \mu \mathrm{g}$ of plasmid in $25 \mathrm{ml}$ of Opti-MEM transfection medium (Life Technologies, CA) was mixed with $5 \mathrm{ml}$ of PEI-MAX $(1.0 \mathrm{mg} / \mathrm{ml})$ in $25 \mathrm{ml}$ of OptiMEM. After $30 \mathrm{~min}$ of incubation, the DNA-PEI-MAX complex was added to 1-L
}

293 F cells. Culture supernatants were harvested 5 days after transfection, clarified by centrifugation at $1126 \times g$ for $22 \mathrm{~min}$, and filtered using a $0.45 \mu \mathrm{m}$ filter (Thermo Scientific). GP $\Delta$ muc proteins were extracted from the supernatants using an mAb114 antibody column or mAb100 antibody column. Bound proteins were eluted three times, each with $5 \mathrm{ml}$ of $0.2 \mathrm{M}$ glycine $(\mathrm{pH} 2.2)$ and neutralized with $0.5 \mathrm{ml}$ of Tris-Base ( $\mathrm{pH} \mathrm{9.0)}$ ), and buffer-exchanged into phosphate-buffered saline (PBS; pH 7.2). Proteins were further purified by SEC on a Superdex 200 Increase 10/300 GL column or HiLoad Superdex 200 16/600 column (GE Healthcare). GP $\Delta$ muc-presenting NPs were produced in ExpiCHO cells (Thermo Fisher). Briefly, ExpiCHO cells were thawed and incubated with ExpiCHO ${ }^{\mathrm{TM}}$ Expression Medium (Thermo Fisher) in a shaker incubator at $37^{\circ} \mathrm{C}$ at $135 \mathrm{rpm}$ with $8 \% \mathrm{CO}_{2}$ When the cells reached a density of $10 \times 10^{6} \mathrm{ml}^{-1}$, ExpiCHO ${ }^{\mathrm{TM}}$ Expression Medium was added to reduce cell density to $6 \times 10^{6} \mathrm{ml}^{-1}$ for transfection. The ExpiFectamine $^{\mathrm{TM}} \mathrm{CHO} /$ plasmid DNA complexes were prepared for $100-\mathrm{ml}$ transfection in ExpiCHO cells following the manufacturer's instructions. For these $\mathrm{NP}$ constructs, $100 \mu \mathrm{g}$ of plasmid and $320 \mu \mathrm{l}$ of ExpiFectamine ${ }^{\mathrm{TM}} \mathrm{CHO}$ reagent were mixed in $7.7 \mathrm{ml}$ of cold OptiPRO ${ }^{\mathrm{sw}}$ medium (Thermo Fisher). After the first feed on day 1, ExpiCHO cells were cultured in a shaker incubator at $33^{\circ} \mathrm{C}$ at 115 rpm with $8 \% \mathrm{CO}_{2}$ according to the Max Titer protocol with an additional feed on day 5 (Thermo Fisher). Culture supernatants were harvested 13-14 days after transfection, clarified by centrifugation at $3724 \times g$ for $25 \mathrm{~min}$, and filtered using a $0.45 \mu \mathrm{m}$ filter (Thermo Fisher). The mAb100 antibody column was used to extract NPs from the supernatants, followed by SEC on a Superose $610 / 300$ GL column. All SEC data were collected using the Unicorn 7.5 software. For GP $\Delta$ muc and GP $\Delta$ muc-presenting NPs, the concentration was determined using $\mathrm{UV}_{280}$ absorbance with theoretical extinction coefficients.

Blue native polyacrylamide gel electrophoresis. EBOV GP $\Delta$ muc and GP $\Delta$ mucpresenting NPs were analyzed by BN-PAGE and stained with Coomassie blue. The proteins were mixed with sample buffer and G250 loading dye and added to a $4-12 \%$ Bis-Tris NativePAGE ${ }^{\mathrm{TM}}$ gel (Life Technologies). BN-PAGE gels were run for $2-2.5 \mathrm{~h}$ at $150 \mathrm{~V}$ using NativePAGE ${ }^{\mathrm{TM}}$ running buffer (Life Technologies) according to the manufacturer's instructions. BN-PAGE images were collected using Image Lab v6.0 software.

Enzyme-linked immunosorbent assay. Each well of a Costar ${ }^{\mathrm{TM}}$ 96-well assay plate (Corning) was first coated with $50 \mu \mathrm{l}$ of PBS containing $0.2 \mu \mathrm{g}$ of appropriate antigens. The plates were incubated overnight at $4{ }^{\circ} \mathrm{C}$, and then washed five times with wash buffer containing PBS and $0.05 \%$ (v/v) Tween 20. Each well was then coated with $150 \mu$ l of blocking buffer consisting of PBS, $40 \mathrm{mg} \mathrm{ml}^{-1}$ blotting-grade blocker (Bio-Rad), and 5\% (v/v) FBS. The plates were incubated with blocking buffer for $1 \mathrm{~h}$ at room temperature, and then washed five times with wash buffer. For antigen binding, antibodies were diluted in blocking buffer to a maximum concentration of $10 \mu \mathrm{g} \mathrm{ml}^{-1}$ followed by a tenfold dilution series. For each antibody dilution, a total of $50 \mu \mathrm{l}$ volume was added to the appropriate wells. For animal sample analysis, plasma/serum was diluted tenfold for mouse and 50-fold for rabbit in blocking buffer and subjected to a tenfold dilution series. For each sample dilution, a total of $50 \mu \mathrm{l}$ volume was added to the wells. Each plate was incubated for $1 \mathrm{~h}$ at room temperature, and then washed five times with PBS containing $0.05 \%$ Tween 20. For antibody binding, a 1:5000 dilution of goat antihuman IgG antibody (Jackson ImmunoResearch Laboratories, Inc), or for animal sample analysis, a 1:2000 dilution of horseradish peroxidase-labeled goat antimouse or anti-rabbit IgG antibody (Jackson ImmunoResearch Laboratories), was then made in wash buffer (PBS containing $0.05 \%$ Tween 20), with $50 \mu \mathrm{l}$ of this diluted secondary antibody added to each well. The plates were incubated with the secondary antibody for $1 \mathrm{~h}$ at room temperature, and then washed five times with PBS containing $0.05 \%$ Tween 20 . Finally, the wells were developed with $50 \mu \mathrm{l}$ of TMB (Life Sciences) for 3-5 min before stopping the reaction with $50 \mu \mathrm{l}$ of $2 \mathrm{~N}$ sulfuric acid. The resulting readouts were measured on a plate reader (PerkinElmer) at a wavelength of $450 \mathrm{~nm}$ and collected using the PerkinElmer 2030 v4.0 software. Notably, the week 2 plasma/serum-GP binding did not reach the plateau (or saturation) to allow for the accurate determination of $\mathrm{EC}_{50}$ titers. Nonetheless, the $\mathrm{EC}_{50}$ values were calculated in GraphPad Prism 8.4.3 and used as a quantitative measure of antibody titers to facilitate comparisons of different vaccine groups at week 2 .

Bio-layer interferometry. The kinetics of GP $\Delta$ muc and GP $\Delta$ muc-presenting NP binding to a panel of 10 antibodies was measured using an Octet RED96 instrument (FortéBio, Pall Life Sciences). All assays were performed with agitation set to $1000 \mathrm{rpm}$ in FortéBio $1 \times$ kinetic buffer. The final volume for all solutions was 200 $\mu \mathrm{l}$ per well. Assays were performed at $30^{\circ} \mathrm{C}$ in solid black $96-$ well plates (Geiger Bio-One). Antibody $\left(5 \mu \mathrm{g} \mathrm{ml}^{-1}\right)$ in $1 \times$ kinetic buffer was loaded onto the surface of anti-human Fc Capture Biosensors (AHC) for GP $\Delta$ muc and of anti-human Fc Quantitation Biosensors (AHQ) for NPs for $300 \mathrm{~s}$. A $60 \mathrm{~s}$ biosensor baseline step was applied prior to analyzing association of the antibody on the biosensor to the antigen in solution for $200 \mathrm{~s}$. A twofold concentration gradient of antigen, starting at $400 \mathrm{nM}$ for GPAmuc trimers, $25 \mathrm{nM}$ for FR NPs, and 10 for E2p/I3-01v9 NPs, was used in a titration series of six. Dissociation of the interaction was followed for $300 \mathrm{~s}$. The correction of baseline drift was performed by subtracting the mean value 
of shifts recorded for a sensor loaded with antibody but not incubated with antigen and for a sensor without antibody but incubated with antigen. The Octet data were processed by FortéBio's data acquisition software v8.2. For GP $\Delta$ muc trimers, experimental data were fitted with the binding equations describing a 2:1 interaction to achieve the optimal fitting and determine the $K_{D}$ values. For GPpresenting NPs, two BLI experiments, one testing six antigen concentrations and the other testing the highest antigen concentration in duplicates, were performed. Binding signals at the highest antigen concentration (mean and standard deviation calculated from three replicates) were used to quantify the effect of multivalent NP display on GP-antibody interactions. Notably, the GP $\Delta$ muc- $\mathrm{WL}^{2} \mathrm{P}^{2}$-foldon trimer was also measured using AHQ biosensors to facilitate comparisons with three NPs that present the GP $\Delta$ muc-WL $\mathrm{WL}^{2} \mathrm{P}^{2}$ trimer multivalently.

Differential scanning calorimetry. Thermal melting curves of wildtype and redesigned GP $\Delta$ muc trimers were obtained with a MicroCal VP-Capillary calorimeter (Malvern). The purified GP $\Delta$ muc protein produced from $293 \mathrm{~F}$ cells was buffer exchanged into $1 \times \mathrm{PBS}$ and concentrated to $27-50 \mu \mathrm{M}$ before analysis by the instrument. Melting was probed at a scan rate of $90^{\circ} \mathrm{C} \mathrm{h}^{-1}$ from 25 to $110^{\circ} \mathrm{C}$. Data processing, including buffer correction, normalization, and baseline subtraction, was conducted using the standardized protocol from Origin 7.0 software.

\section{Protein production, crystallization, and data collection. Two Zaire EBOV}

GP $\Delta$ muc-foldon constructs, one with the W615L mutation and the L extension (to residue 637) and the other with an additional T577P mutation, were expressed in HEK293S cells. The expressed GP was purified using an mAB100 antibody column followed by SEC on a HiLoad Superdex 200 16/600 column (GE Healthcare). PBS $(\mathrm{pH}$ 7.2) was used as the gel filtration buffer during the purification process. The freshly purified GP protein was used for crystallization experiments using the sitting drop vapor diffusion method on our automated CrystalMation ${ }^{\mathrm{TM}}$ robotic system (Rigaku) at both 4 and $20^{\circ} \mathrm{C}$ at The Scripps Research Institute (TSRI) ${ }^{99}$ EBOV GP was concentrated to $\sim 10 \mathrm{mg} / \mathrm{ml}$ in $50 \mathrm{mM}$ Tris- $\mathrm{HCl}$, pH 8.0. The reservoir solution contained $12 \%(\mathrm{w} / \mathrm{v})$ PEG 6000 and $0.1 \mathrm{M}$ sodium citrate, $\mathrm{pH}$ 4.5. Diffraction-quality crystals were obtained after 2 weeks at $20^{\circ} \mathrm{C}$. The EBOV GP crystals were cryoprotected with well solution augmented with $25 \%$ glycerol, mounted in a nylon loop and flash cooled in liquid nitrogen. Diffraction data were collected for crystals of GP $\Delta$ muc-WL ${ }^{2}$-foldon and GP $\Delta$ muc-WL $\mathrm{WL}^{2} \mathrm{P}^{2}$-foldon at Advanced Photon Source (APS) beamline 23ID-D and Stanford Synchrotron Radiation Lightsource (SSRL) beamline 12-2, at 2.3 and $3.2 \AA$ resolution, respectively. The diffraction data sets were processed with HKL-2000 ${ }^{100}$. The crystal data were indexed in R32 and P321 space groups with cell dimensions of GP $\Delta$ muc$\mathrm{WL}^{2}$-foldon $a=b=114.58 \AA$ and $c=312.38 \AA$ and GP $\Delta$ muc-WL ${ }^{2} \mathrm{P}^{2}$-foldon $a=$ $b=114.06 \AA$ and $c=136.22 \AA$, respectively (Table S2). The overall completeness of the two datasets was 96.4 and $99.9 \%$.

Structure determination and refinement. The structures of EBOV GP were determined by molecular replacement (MR) using Phaser ${ }^{101}$ from the CCP4i suite $^{102}$ with the coordinates of Zaire Ebola GP (PDB ID: 5JQ3) and the program MOLREP $^{103}$. The polypeptide chains were manually adjusted into electron density using Coot ${ }^{104}$, refined with Refmac 5.8 $8^{105}$, and validated using MolProbity ${ }^{106}$. The final $R_{\text {work }}$ and $R_{\text {free }}$ values for the refined structures are $20.0 \%$ and $23.7 \%$, and $27.7 \%$ and $32.2 \%$, for GP $\Delta$ muc-WL $\mathrm{WL}^{2}$-foldon and GP $\Delta$ muc-WL $\mathrm{WL}^{2} \mathrm{P}^{2}$-foldon, respectively. The data processing and refinement statistics are compiled in Table S2. Structural images shown in Fig. 3 and Supplementary Figs. 3-5 were generated using PyMOL v2.3.4 software.

Electron microscopy (EM) assessment of nanoparticle constructs. The initial EM analysis of EBOV GP $\triangle$ Muc NPs was conducted at the Core Microscopy Facility at The Scripps Research Institute. Briefly, NP samples were prepared at a concentration of $0.01 \mathrm{mg} / \mathrm{ml}$. Carbon-coated copper grids ( $400 \mathrm{mesh}$ ) were glowdischarged, and $8 \mu$ of each sample was adsorbed for $2 \mathrm{~min}$. Excess sample was wicked away, and grids were negatively stained with $2 \%$ uranyl formate for $2 \mathrm{~min}$. Excess stain was wicked away, and the grids were allowed to dry. Samples were analyzed at $80 \mathrm{kV}$ with a Talos L120C transmission electron microscope (Thermo Fisher), and images were acquired with a CETA 16 M CMOS camera. Further EM analysis was conducted at the Hazen facility at The Scripps Research Institute. NP samples were diluted to $\sim 0.02 \mathrm{mg} / \mathrm{ml}$ and added onto carbon-coated copper 400 mesh grids (Electron Microscopy Sciences) that had been plasma cleaned for $10 \mathrm{~s}$ with $\mathrm{Ar} / \mathrm{O} 2$. After blotting to remove excess sample, grids were stained with $3 \mu \mathrm{l}$ of $2 \%(\mathrm{w} / \mathrm{v})$ uranyl formate for $60 \mathrm{~s}$ and blotted again to remove excess stain. Negative-stain images were collected on a $120 \mathrm{KeV}$ Tecnai Spirit equipped with an Eagle $4 \mathrm{~K}$ charge-coupled device camera (FEI). Micrographs were collected using Leginon ${ }^{107}$ and processed using cryoSPARC $\mathrm{v}^{108}$. Micrographs were CTF corrected, and particles were picked manually and extracted for two-dimensional classification.

Animal immunization and sample collection. Similar immunization protocols were reported in our previous HIV-1 and HCV studies ${ }^{55,65}$. Briefly, the Institutional Animal Care and Use Committee (IACUC) guidelines were followed with animal subjects tested in the immunization study. Six-to-eight-week-old female
BALB/c mice were purchased from The Jackson Laboratory and housed in ventilated cages in environmentally controlled rooms at The Scripps Research Institute, in compliance with an approved IACUC protocol and AAALAC (Association for Assessment and Accreditation of Laboratory Animal Care) international guidelines. The vivarium was maintained at $22^{\circ} \mathrm{C}$ with a 13 -h light/11-h dark cycle (lights on at 6:00 a.m. and off at 7:00 p.m.) and 40-50\% humidity, which might be reduced to $30-40 \%$ during the winter. The mice were immunized at weeks $0,3,6$, and 9 with $200 \mu \mathrm{l}$ of antigen/adjuvant mix containing $50 \mu \mathrm{g}$ of vaccine antigen and $100 \mu \mathrm{l}$ of adjuvant, AddaVax or Adju-Phos (InvivoGen), via the intraperitoneal (i.p.) route. Of note, $20 \mu \mathrm{g}$ instead of $50 \mu \mathrm{g}$ of mAb100-purified I3-01v9 protein was used in mouse immunization due to its low yield. Blood was collected 2 weeks after each immunization. All bleeds were performed through the retro-orbital sinus using heparinized capillary tubes into EDTA-coated tubes. Samples were diluted with an equal volume of PBS and then overlaid on $4.5 \mathrm{ml}$ of Ficoll in a $15 \mathrm{ml}$ SepMate ${ }^{\mathrm{TM}}$ tube (STEMCELL Technologies) and spun at $300 \times g$ for $10 \mathrm{~min}$ at $20^{\circ} \mathrm{C}$ to separate plasma and cells. The plasma was heat inactivated at $56^{\circ} \mathrm{C}$ for $30 \mathrm{~min}$, spun at $300 \times g$ for $10 \mathrm{~min}$, and sterile filtered. The cells were washed once in PBS and then resuspended in $1 \mathrm{ml}$ of ACK Red Blood Cell lysis buffer (Lonza). After washing with PBS, peripheral blood mononuclear cells (PBMCs) were resuspended in $2 \mathrm{ml}$ of Bambanker Freezing Media (Lymphotec). Spleens were also harvested and ground against a $70-\mu \mathrm{m}$ cell strainer (BD Falcon) to release splenocytes into a cell suspension. Splenocytes were centrifuged, washed in PBS, treated with $5 \mathrm{ml}$ of ACK lysing buffer (Lonza), and frozen with $3 \mathrm{ml}$ of Bambanker freezing media. Purified mouse IgGs at w11 were obtained using a $0.2-\mathrm{ml}$ protein $\mathrm{G}$ spin kit (Thermo Scientific) following the manufacturer's instructions and assessed in pseudovirus neutralization assays. Rabbit immunization and blood sampling were performed under a subcontract at ProSci (San Diego, CA) under the IACUC protocol number APF-1A and related amendments (10/01/2018 through 10/01/2021). Five groups of female New Zealand White rabbits, four rabbits per group, were intramuscularly (i. $\mathrm{m}$.) immunized with $50 \mu \mathrm{g}$ of vaccine antigen formulated in $250 \mu \mathrm{l}$ of adjuvant, AddaVax or Adju-Phos (InvivoGen), with a total volume of $500 \mu$, at w0, w3, w6, and w9. Of note, $20 \mu \mathrm{g}$ of mAb100/SEC-purified I3-01v9 NP was used for rabbit immunization. Blood samples, $20 \mathrm{ml}$ each time, were collected from the auricular artery at day 0 (Pre), w2, w5, w8, and w11. More than $100 \mathrm{ml}$ of blood was taken at $\mathrm{w} 13$, via cardiac puncture, for PBMC isolation. Serum samples were heat inactivated for ELISA binding assays, and purified rabbit IgGs from different time points were assessed in pseudovirus neutralization assays.

Pseudovirus neutralization assay. The ebolavirus pseudoparticle (ebolavirus-pp) neutralization assay ${ }^{12}$ was performed to assess the neutralizing activity of previously reported mAbs and vaccine-induced antibody responses in mice and rabbits. Ebolavirus-pps were generated by the co-transfection of HEK293T cells with the HIV-1 pNL4-3.lucR-E- plasmid (NIH AIDS reagent program: https://www. aidsreagent.org/) and the expression plasmid encoding the GP gene of an EBOV Makona strain (GenBank accession no. KJ660346) or BDBV Uganda strain (GenBank accession no. KR063673) at a 4:1 ratio by lipofectamine 3000 (Thermo Fisher). After $48-72 \mathrm{~h}$, ebolavirus-pps were collected from the supernatant by centrifugation at $3724 \times \mathrm{g}$ for $10 \mathrm{~min}$, aliquoted, and stored at $-80^{\circ} \mathrm{C}$ until use. The mAbs at a starting concentration of $10 \mu \mathrm{g} / \mathrm{ml}$, or purified IgGs at a starting concentration of $300 \mu \mathrm{g} / \mathrm{ml}$ for mouse and $1000 \mu \mathrm{g} / \mathrm{ml}$ for rabbit, were mixed with the supernatant containing ebolavirus-pps and incubated for $1 \mathrm{~h}$ at $37^{\circ} \mathrm{C}$ in white solid-bottom 96-well plates (Corning). Based on recent studies on EBOV infectivity in various cell lines $72,109,293 \mathrm{~T}$ cells or TZM-bl cells were used for ebolavirus-pp neutralization assays. Briefly, HEK293T cells or TZM-bl cells at $1 \times 10^{4}$ were added to each well, and the plate was incubated at $37^{\circ} \mathrm{C}$ for $48 \mathrm{~h}$. After incubation, overlying media was removed, and cells were lysed. The firefly luciferase signal from infected cells was determined using the Bright-Glo Luciferase Assay System (Promega) according to the manufacturer's instructions. Data were retrieved from a BioTek microplate reader with Gen 5 software. The average background luminescence from a series of uninfected wells was subtracted from each well, and neutralization curves were generated using GraphPad Prism 8.4.3, in which values from wells were compared against a well containing ebolavirus-pp only. The same HIV-1 vectors pseudotyped with the murine leukemia virus (MLV) Env gene, termed MLV-pps, were produced in $293 \mathrm{~T}$ cells and included in the neutralization assays as a negative control. Because non-NAb c13C6 exhibited enhanced MLV-pp infection, the MLV-pp assay was also used to detect and quantify the glycan capdirected non-NAb response in immunized animal samples.

Bulk sorting of EBOV GPAmuc-specific mouse splenic B cells. Spleens were harvested from mice 15 days after the last immunization, and the cell suspension was prepared. Dead cells were excluded by staining with the Fixable Aqua Dead Cell Stain kit (Thermo Fisher L34957). FcyIII (CD16) and FcyII (CD32) receptors were blocked by adding $20 \mu \mathrm{l}$ of $2.4 \mathrm{G} 2 \mathrm{mAb}$ (BD Pharmigen, catalog no. N553142). The cells were then incubated with $10 \mu \mathrm{g} / \mathrm{ml}$ of a biotinylated GP $\Delta$ muc-WL $\mathrm{WL}^{2} \mathrm{P}^{2}$ 1TD0-Avi trimer. Briefly, the probe was generated by biotinylation of the $\mathrm{GP} \Delta$ muc-WL $\mathrm{WL}^{2} \mathrm{P}^{2}-1 \mathrm{TD} 0$-Avi trimer using biotin ligase BirA according to the manufacturer's instructions (Avidity). Biotin excess was removed by SEC on a Superdex 200 10/300 column (GE Healthcare). In the SEC profile, the Avi-tagged GP $\Delta$ muc trimer peak was centered at $10.0-11.0 \mathrm{ml}$, whereas a broader peak of biotin ligase was found at $20 \mathrm{ml}$. Cells and biotinylated proteins were incubated for 
$5 \mathrm{~min}$ at $4{ }^{\circ} \mathrm{C}$, followed by the addition of $2.5 \mu \mathrm{l}$ of anti-mouse IgG fluorescently labeled with FITC (Jackson ImmunoResearch, catalog no. 115-095-071) and incubated for $15 \mathrm{~min}$ at $4^{\circ} \mathrm{C}$. Finally, $5 \mu \mathrm{l}$ of premium-grade allophycocyanin (APC)-labeled streptavidin was added to cells and incubated for $15 \mathrm{~min}$ at $4{ }^{\circ} \mathrm{C}$. In each step, the cells were washed with PBS and the sorting buffer was $0.5 \mathrm{ml}$ of FACS buffer. FITC ${ }^{+} \mathrm{APC}^{+} \mathrm{GP} \Delta$ muc-specific B cells were sorted using MoFloAstrios into one well of a 96-well plate with $20 \mu \mathrm{l}$ of lysis buffer. Gating strategies used in antigen-specific mouse B-cell sorting are exemplified by the flowchart in Fig. S9b. Briefly, antigen-specific mouse splenic B cells were isolated by gating on single cells that were live/dead marker negative, mouse IgG positive, and biotinylated EBOV GP positive. Flow cytometry data were collected using the Summit v6.3 software.

NGS and bioinformatics analysis of mouse B cells. Previously, a $5^{\prime}$-rapid amplification of cDNA ends (RACE)-polymerase chain reaction (PCR) protocol was reported for the unbiased sequencing of mouse B-cell repertoires ${ }^{65}$. In this study, this protocol was applied to analyze bulk-sorted, GP-specific mouse splenic B cells. Briefly, 5'-RACE CDNA was obtained from bulk-sorted splenic B cells of each mouse with the SMART-Seq v4 Ultra Low Input RNA Kit for Sequencing (TaKaRa). The IgG PCRs were set up with Platinum Taq High-Fidelity DNA Polymerase (Life Technologies) in a total volume of $50 \mu \mathrm{l}$, with $5 \mu \mathrm{l}$ of cDNA as the template, $1 \mu \mathrm{l}$ of $5^{\prime}$-RACE primer, and $1 \mu \mathrm{l}$ of $10 \mu \mathrm{M}$ reverse primer. The $5^{\prime}$-RACE primer contained a PGM/S5 P1 adaptor, while the reverse primer contained a PGM/S5 A adaptor. We adapted the mouse $3^{\prime}-\mathrm{C}_{\gamma} 1-3 / 3^{\prime}-\mathrm{C}_{\mu}$ inner primers and $3^{\prime}-$ $\mathrm{mC}_{\mathrm{K}}$ outer primer as reverse primers for the $5^{\prime}$-RACE PCR processing of heavy and light ( $\kappa$ ) chains (Table S1). A total of 25 cycles of PCR was performed, and the expected PCR products (500-600 bp) were gel purified (Qiagen). NGS was performed on the Ion S5 GeneStudio system. Briefly, heavy and light ( $\mathrm{\kappa}$ ) chain libraries from the same mouse were quantitated using a Qubit ${ }^{\circledR} 2.0$ Fluorometer with Qubit ${ }^{\circledR}$ dsDNA HS Assay Kit and then mixed at a 3:1 ratio before being pooled with antibody libraries of other mice at an equal ratio for sequencing. Template preparation and (Ion 530) chip loading were performed on Ion Chef using the Ion $520 / 530$ Ext Kit, followed by sequencing on the Ion S5 system with default settings. The mouse Antibodyomics pipeline ${ }^{65}$ was used to process raw NGS data and derive quantitative profiles for germline gene usage, degree of SHM, and H/KCDR3 loop length.

Statistics and reproducibility. SEC was performed for all GP/NP constructs at least once for in vitro characterization and multiple times during protein production for animal studies. Representative SEC profiles were selected for comparison. BN-PAGE was performed for all GP/NP constructs at least once during screening, with selected constructs run on the same gel to facilitate visual comparison. DSC was performed up to three times to validate key thermal parameters and thermographs. Negative-stain EM was performed routinely for all NP constructs during in vitro characterization and protein production for animal studies. All ELISA binding assays were performed in duplicates. Due to the limited availability of purified mouse IgG samples, ebolavirus-pp neutralization assays were performed without duplicates. For the panel of 10 representative antibodies and purified rabbit IgG samples, ebolavirus-pp neutralization assays were performed in duplicates. An unpaired two-tailed $t$ test was performed in GraphPad Prism 8.4.3 to determine $P$ values in the analysis of binding antibody response and mouse B-cell repertoires. The level of statistical significance is indicated as: ${ }^{*} P<0.05$; ${ }^{* *} P<0.01$; $* * * P<0.001 ; * * * * P<0.0001$.

Reporting summary. Further information on research design is available in the Nature Research Reporting Summary linked to this article.

\section{Data availability}

The X-ray crystallographic coordinates for two rationally redesigned GP $\Delta$ muc structures in this study have been deposited in the Protein Data Bank (PDB, https://www.rcsb.org/), under accession codes 7JPI (https://doi.org/10.2210/pdb7jpi/pdb) and 7JPH (https://doi. org/10.2210/pdb7jph/pdb). The mouse B-cell NGS datasets have been deposited in the NIH Sequence Read Archive (SRA, https://www.ncbi.nlm.nih.gov/sra), with the identifier PRJNA718964. The authors declare that the data supporting the findings of this study are available within the article and its Supplementary Information files. Source data are provided with this paper.

Received: 27 February 2021; Accepted: 6 April 2021; Published online: 11 May 2021

\section{References}

1. Kuhn, J. H. et al. Virus nomenclature below the species level: a standardized nomenclature for filovirus strains and variants rescued from cDNA. Arch. Virol. 159, 1229-1237 (2014).
2. Paessler, S. \& Walker, D. H. Pathogenesis of the viral hemorrhagic fevers. Annu. Rev. Pathol. 8, 411-440 (2013).

3. Malvy, D., McElroy, A. K., de Clerck, H., Gunther, S. \& van Griensven, J. Ebola virus disease. Lancet 393, 936-948 (2019).

4. Ebola outbreak 2014-2016 (https://www.who.int/csr/disease/ebola/en/).

5. Ebola situation reports: Democratic Republic of the Congo (https://www.who. int/ebola/situation-reports/drc-2018/en/).

6. Cardile, A. P., Downey, L. G., Wiseman, P. D., Warren, T. K. \& Bavari, S. Antiviral therapeutics for the treatment of Ebola virus infection. Curr. Opin. Pharmacol. 30, 138-143 (2016).

7. Cardile, A. P., Warren, T. K., Martins, K. A., Reisler, R. B. \& Bavari, S. Will there be a cure for Ebola? Annu. Rev. Pharmacol. Toxicol. 57, 329-348 (2017).

8. Mire, C. E. \& Geisbert, T. W. Neutralizing the threat: pan-ebolavirus antibodies close the loop. Trends Mol. Med. 23, 669-671 (2017).

9. King, L. B., Milligan, J. C., West, B. R., Schendel, S. L. \& Saphire, E. O. Achieving cross-reactivity with pan-ebolavirus antibodies. Curr. Opin. Virol. 34, 140-148 (2019)

10. Qiu, X. G. et al. Reversion of advanced Ebola virus disease in nonhuman primates with ZMapp. Nature 514, 47-53 (2014).

11. Davey, R. T. et al. A randomized, controlled trial of ZMapp for Ebola virus infection. N. Engl. J. Med. 375, 1448-1456 (2016).

12. Corti, D. et al. Protective monotherapy against lethal Ebola virus infection by a potently neutralizing antibody. Science 351, 1339-1342 (2016).

13. Gaudinski, M. R. et al. Safety, tolerability, pharmacokinetics, and immunogenicity of the therapeutic monoclonal antibody mAb114 targeting Ebola virus glycoprotein (VRC 608): an open-label phase 1 study. Lancet 393 889-898 (2019).

14. Zhu, F. C. et al. Safety and immunogenicity of a recombinant adenovirus type5 vector-based Ebola vaccine in healthy adults in Sierra Leone: a single-centre, randomised, double-blind, placebo-controlled, phase 2 trial. Lancet 389 , 621-628 (2017)

15. Kennedy, S. B. et al. Phase 2 placebo-controlled trial of two vaccines to prevent Ebola in liberia. N. Engl. J. Med. 377, 1438-1447 (2017).

16. Henao-Restrepo, A. M. et al. Efficacy and effectiveness of an rVSV-vectored vaccine in preventing Ebola virus disease: final results from the Guinea ring vaccination, open-label, cluster-randomised trial (Ebola Ça Suffit!). Lancet 389, 505-518 (2017).

17. Sarwar, U. N. et al. Safety and immunogenicity of DNA vaccines encoding ebolavirus and marburgvirus wild-type glycoproteins in a phase I clinical trial. J. Infect. Dis. 211, 549-557 (2015).

18. Jones, S. M. et al. Live attenuated recombinant vaccine protects nonhuman primates against Ebola and Marburg viruses. Nat. Med. 11, 786-790 (2005).

19. Qiu, X. G. et al. Mucosal immunization of cynomolgus macaques with the VSV delta G/ZEBOVGP vaccine stimulates strong Ebola GP-specific immune responses. PLoS One 4, e5547 (2009).

20. Geisbert, T. W. et al. Single-injection vaccine protects nonhuman primates against infection with Marburg virus and three species of Ebola virus. J. Virol. 83, 7296-7304 (2009).

21. Geisbert, T. W. et al. Vesicular stomatitis virus-based Ebola vaccine is welltolerated and protects immunocompromised nonhuman primates. PLoS Pathog. 4, e1000225 (2008)

22. Lévy, Y. et al. Prevention of Ebola virus disease through vaccination: where we are in 2018. Lancet 392, 787-790 (2018).

23. Marzi, A. et al. Antibodies are necessary for rVSV/ZEBOV-GP-mediated protection against lethal Ebola virus challenge in nonhuman primates. Proc. Natl Acad. Sci. USA 110, 1893-1898 (2013).

24. McWilliams, I. L. et al. Pseudovirus rVSV delta G-ZEBOV-GP infects neurons in retina and CNS, causing apoptosis and neurodegeneration in neonatal mice. Cell Rep. 26, 1718-1726.e1714 (2019).

25. Kuzmina, N. A. et al. Antibody-dependent enhancement of Ebola virus infection by human antibodies isolated from survivors. Cell Rep. 24, 1802-1815.e1805 (2018).

26. Lee, J. E. \& Saphire, E. O. Ebolavirus glycoprotein structure and mechanism of entry. Future Virol. 4, 621-635 (2009).

27. McElroy, A. K. et al. Human Ebola virus infection results in substantial immune activation. Proc. Natl Acad. Sci. USA 112, 4719-4724 (2015).

28. Davis, C. W. et al. Longitudinal analysis of the human B cell response to Ebola virus infection. Cell 177, 1566-1582.e1517 (2019).

29. Williamson, L. E. et al. Early human B cell response to Ebola virus in four US survivors of infection. J. Virol. 93, e01439-01418 (2019).

30. Saphire, E. O. \& Aman, M. J. Feverish quest for Ebola immunotherapy: straight or cocktail? Trends Microbiol. 24, 684-686 (2016).

31. Flyak, A. I. et al. Mechanism of human antibody-mediated neutralization of Marburg virus. Cell 160, 893-903 (2015).

32. King, L. B. et al. The marburgvirus-neutralizing human monoclonal antibody MR191 targets a conserved site to block virus receptor binding. Cell Host Microbe 23, 101-109.e104 (2018). 
33. Fusco, M. L. et al. Protective mAbs and cross-reactive mAbs raised by immunization with engineered Marburg virus GPs. PLoS Pathog. 11, e1005016 (2015)

34. Keck, Z. Y. et al. Macaque monoclonal antibodies targeting novel conserved epitopes within filovirus glycoprotein. J. Virol. 90, 279-291 (2016).

35. Howell, K. A. et al. Antibody treatment of Ebola and Sudan virus infection via a uniquely exposed epitope within the glycoprotein receptor-binding site. Cell Rep. 15, 1514-1526 (2016)

36. Zhao, X. L. et al. Immunization-elicited broadly protective antibody reveals ebolavirus fusion loop as a site of vulnerability. Cell 169, 891-904.e815 (2017).

37. Bornholdt, Z. A. et al. Isolation of potent neutralizing antibodies from a survivor of the 2014 Ebola virus outbreak. Science 351, 1078-1083 (2016).

38. Wec, A. Z. et al. Antibodies from a human survivor define sites of vulnerability for broad protection against ebolaviruses. Cell 169, 878-890 (2017).

39. Gilchuk, P. et al. Multifunctional pan-ebolavirus antibody recognizes a site of broad vulnerability on the ebolavirus glycoprotein. Immunity 49, 363-374. e310 (2018).

40. Lee, J. E. et al. Structure of the Ebola virus glycoprotein bound to an antibody from a human survivor. Nature 454, 177-182 (2008)

41. Dias, J. M. et al. A shared structural solution for neutralizing ebolaviruses. Nat. Struct. Mol. Biol. 18, 1424-1427 (2011).

42. Misasi, J. et al. Structural and molecular basis for Ebola virus neutralization by protective human antibodies. Science 351, 1343-1346 (2016).

43. Bornholdt, Z. A. et al. Host-primed Ebola virus GP exposes a hydrophobic NPC1 receptor-binding pocket, revealing a target for broadly neutralizing antibodies. mBio 7, e02154-15 (2016).

44. Murin, C. D. et al. Structures of protective antibodies reveal sites of vulnerability on Ebola virus. Proc. Natl Acad. Sci. USA 111, 17182-17187 (2014).

45. Pallesen, J. et al. Structures of Ebola virus GP and sGP in complex with therapeutic antibodies. Nat. Microbiol. 1, 16128 (2016).

46. Flyak, A. I. et al. Cross-reactive and potent neutralizing antibody responses in human survivors of natural ebolavirus infection. Cell 164, 392-405 (2016).

47. Gilchuk, P. et al. Analysis of a therapeutic antibody cocktail reveals determinants for cooperative and broad ebolavirus neutralization. Immunity 52, 388-403.e312 (2020).

48. Saphire, E. O. et al. Systematic analysis of monoclonal antibodies against Ebola virus GP defines features that contribute to protection. Cell 174, 938-952.e913 (2018).

49. Walker, L. M. \& Burton, D. R. Rational antibody-based HIV-1 vaccine design: current approaches and future directions. Curr. Opin. Immunol. 22, 358-366 (2010).

50. Haynes, B. F. \& Mascola, J. R. The quest for an antibody-based HIV vaccine. Immunol. Rev. 275, 5-10 (2017)

51. Sanders, R. W. \& Moore, J. P. Native-like Env trimers as a platform for HIV-1 vaccine design. Immunol. Rev. 275, 161-182 (2017).

52. Ward, A. B. \& Wilson, I. A. The HIV-1 envelope glycoprotein structure: nailing down a moving target. Immunol. Rev. 275, 21-32 (2017).

53. White, J. M., Delos, S. E., Brecher, M. \& Schornberg, K. Structures and mechanisms of viral membrane fusion proteins: multiple variations on a common theme. Crit. Rev. Biochem. Mol. Biol. 43, 189-219 (2008).

54. Harrison, S. C. Viral membrane fusion. Virology 479, 498-507 (2015).

55. He, L. et al. HIV-1 vaccine design through minimizing envelope metastability. Sci. Adv. 4, aau6769 (2018).

56. Kong, L. et al. Uncleaved prefusion-optimized gp140 trimers derived from analysis of HIV-1 envelope metastability. Nat. Commun. 7, 12040 (2016).

57. Aldon, Y. et al. Rational design of DNA-expressed stabilized native-like HIV-1 envelope trimers. Cell Rep. 24, 3324-3338.e3325 (2018)

58. Brouwer, P. J. M. \& Sanders, R. W. Presentation of HIV-1 envelope glycoprotein trimers on diverse nanoparticle platforms. Curr. Opin. HIV AIDS 14, 302-308 (2019).

59. Kulp, D. W. \& Xu, Z. Protein engineering and particulate display of B-cell epitopes to facilitate development of novel vaccines. Curr. Opin. Immunol. 59, 49-56 (2019)

60. Warfield, K. L. et al. Ebola virus-like particle-based vaccine protects nonhuman primates against lethal Ebola virus challenge. J. Infect. Dis. 196, S430-S437 (2007).

61. Swenson, D. L. et al. Virus-like particles exhibit potential as a pan-filovirus vaccine for both Ebola and Marburg viral infections. Vaccine 23, 3033-3042 (2005).

62. Warfield, K. L. et al. Ebola virus-like particles protect from lethal Ebola virus infection. Proc. Natl Acad. Sci. USA 100, 15889-15894 (2003).

63. Feldmann, H., Feldmann, F. \& Marzi, A. Ebola: Lessons on vaccine development. Annu. Rev. Microbiol. 72, 423-446 (2018).

64. He, L. et al. Presenting native-like trimeric HIV-1 antigens with selfassembling nanoparticles. Nat. Commun. 7, 12041 (2016).
65. He, L. L. et al. Proof of concept for rational design of hepatitis C virus E2 core nanoparticle vaccines. Sci. Adv. 6, eaaz6225 (2020).

66. Zhao, Y. G. et al. Toremifene interacts with and destabilizes the Ebola virus glycoprotein. Nature 535, 169-172 (2016).

67. Pugach, P. et al. A native-like SOSIP.664 trimer based on an HIV-1 subtype B env gene. J. Virol. 89, 3380-3395 (2015).

68. Sanders, R. W. et al. A next-generation cleaved, soluble HIV-1 Env trimer, BG505 SOSIP.664 gp140, expresses multiple epitopes for broadly neutralizing but not non-neutralizing antibodies. PLoS Pathog. 9, e1003618 (2013).

69. Sliepen, K. et al. Presenting native-like HIV-1 envelope trimers on ferritin nanoparticles improves their immunogenicity. Retrovirology 12, 82 (2015).

70. Lyumkis, D. et al. Cryo-EM structure of a fully glycosylated soluble cleaved HIV-1 envelope trimer. Science 342, 1484-1490 (2013).

71. Julien, J.-P. et al. Crystal structure of a soluble cleaved HIV-1 envelope trimer Science 342, 1477-1483 (2013).

72. Shaikh, F. et al. Structure-based in silico screening identifies a potent ebolavirus inhibitor from a traditional Chinese medicine library. J. Med. Chem. 62, 2928-2937 (2019).

73. Ren, J. S., Zhao, Y. G., Fry, E. E. \& Stuart, D. I. Target identification and mode of action of four chemically divergent drugs against ebolavirus infection. $J$. Med. Chem. 61, 724-733 (2018).

74. King, L. B. et al. Cross-reactive neutralizing human survivor monoclonal antibody BDBV223 targets the ebolavirus stalk. Nat. Commun. 10, 1788 (2019).

75. Dolnik, O. et al. Ectodomain shedding of the glycoprotein GP of Ebola virus. EMBO J. 23, 2175-2184 (2004).

76. Maruyama, T. et al. Ebola virus can be effectively neutralized by antibody produced in natural human infection. J. Virol. 73, 6024-6030 (1999).

77. Janus, B. M. et al. Structural basis for broad neutralization of ebolaviruses by an antibody targeting the glycoprotein fusion loop. Nat. Commun. 9, 3934 (2018).

78. Yin, H. S., Wen, X. L., Paterson, R. G., Lamb, R. A. \& Jardetzky, T. S. Structure of the parainfluenza virus $5 \mathrm{~F}$ protein in its metastable, prefusion conformation. Nature 439, 38-44 (2006).

79. Liu, J., Shu, W., Fagan, M. B., Nunberg, J. H. \& Lu, M. Structural and functional analysis of the HIV gp41 core containing an Ile573 to Thr substitution: Implications for membrane fusion. Biochemistry 40, 2797-2807 (2001).

80. Malashkevich, V. N. et al. Core structure of the envelope glycoprotein GP2 from Ebola virus at 1.9-Å resolution. Proc. Natl Acad. Sci. USA 96, 2662-2667 (1999).

81. Koellhoffer, J. F. et al. Crystal structure of the Marburg virus GP2 core domain in its postfusion conformation. Biochemistry 51, 7665-7675 (2012).

82. Harrison, J. S., Koellhoffer, J. F., Chandran, K. \& Lai, J. R. Marburg virus glycoprotein GP2: pH-dependent stability of the ectodomain $\alpha$-helical bundle. Biochemistry 51, 2515-2525 (2012).

83. Wang, Q. et al. Stabilized diverse HIV-1 envelope trimers for vaccine design Emerg. Microbes Infect. 9, 775-786 (2020).

84. Rutten, L. et al. Structure-based design of prefusion-stabilized filovirus glycoprotein trimers. Cell Rep. 30, 4540-4550.e4543 (2020).

85. McLellan, J. S. et al. Structure-based design of a fusion glycoprotein vaccine for respiratory syncytial virus. Science 342, 592-598 (2013).

86. Hastie, K. M. et al. Structural basis for antibody-mediated neutralization of Lassa virus. Science 356, 923-928 (2017).

87. Leaver-Fay, A. et al. ROSETTA3: An object-oriented software suite for the simulation and design of macromolecles. Methods Enzymol. 487, 545-574 (2011).

88. Bachmann, M. F. \& Jennings, G. T. Vaccine delivery: a matter of size, geometry, kinetics and molecular patterns. Nat. Rev. Immunol. 10, 787-796 (2010).

89. Marcandalli, J. et al. Induction of potent neutralizing antibody responses by a designed protein nanoparticle vaccine for respiratory syncytial virus. Cell 176, 1420-1431.e1417 (2019).

90. Bale, J. B. et al. Accurate design of megadalton-scale two-component icosahedral protein complexes. Science 353, 389-394 (2016).

91. Winstead, C. R., Zhai, S. K., Sethupathi, P. \& Knight, K. L. Antigen-induced somatic diversification of rabbit IgH genes: gene conversion and point mutation. J. Immunol. 162, 6602-6612 (1999).

92. Morris, C. D. et al. Differential antibody responses to conserved HIV-1 neutralizing epitopes in the context of multivalent scaffolds and native-like gp140 trimers. mBio 8, e00036-00017 (2017).

93. Martins, K. A., Jahrling, P. B., Bavari, S. \& Kuhn, J. H. Ebola virus disease candidate vaccines under evaluation in clinical trials. Expert Rev. Vaccines 15 1101-1112 (2016)

94. Rijal, P. et al. Therapeutic monoclonal antibodies for Ebola virus infection derived from vaccinated humans. Cell Rep. 27, 172-186.e177 (2019).

95. Ewer, K. et al. A monovalent chimpanzee adenovirus Ebola vaccine boosted with MVA. N. Engl. J. Med. 374, 1635-1646 (2016). 
96. Meyer, M., Malherbe, D. C. \& Bukreyev, A. Can Ebola virus vaccines have universal immune correlates of protection? Trends Microbiol. 27, 8-16 (2019).

97. Bruun, T. U. J., Andersson, A. M. C., Draper, S. J. \& Howarth, M. Engineering a rugged nanoscaffold to enhance plug-and-display vaccination. ACS Nano 12, 8855-8866 (2018).

98. Zakeri, B. et al. Peptide tag forming a rapid covalent bond to a protein, through engineering a bacterial adhesin. Proc. Natl Acad. Sci. USA 109, E690-E697 (2012).

99. Elsliger, M.-A. et al. The JCSG high-throughput structural biology pipeline. Acta Crystallogr. Sect. F. Struct. Biol. Cryst. Commun. 66, 1137-1142 (2010).

100. Otwinowski, Z. \& Minor, W. Processing of X-ray diffraction data collected in oscillation mode. Methods Enzymol. 276, 307-326 (1997).

101. McCoy, A. J. et al. Phaser crystallographic software. J. Appl. Crystallogr. 40 658-674 (2007).

102. Potterton, E., Briggs, P., Turkenburg, M. \& Dodson, E. A graphical user interface to the CCP4 program suite. Acta Crystallogr. D. Biol. Crystallogr. 59, 1131-1137 (2003).

103. Vagin, A. \& Teplyakov, A. Molecular replacement with MOLREP. Acta Crystallogr. D. Biol. Crystallogr. 66, 22-25 (2010).

104. Emsley, P., Lohkamp, B., Scott, W. G. \& Cowtan, K. Features and development of Coot. Acta Crystallogr. D. Biol. Crystallogr. 66, 486-501 (2010).

105. Vagin, A. A. et al. REFMAC5 dictionary: organization of prior chemical knowledge and guidelines for its use. Acta Crystallogr. D. Biol. Crystallogr. 60, 2184-2195 (2004).

106. Chen, V. B. et al. MolProbity: all-atom structure validation for macromolecular crystallography. Acta Crystallogr. D. Biol. Crystallogr. 66 12-21 (2010)

107. Suloway, C. et al. Automated molecular microscopy: The new Leginon system. J. Struct. Biol. 151, 41-60 (2005).

108. Punjani, A., Rubinstein, J. L., Fleet, D. J. \& Brubaker, M. A. cryoSPARC: algorithms for rapid unsupervised cryo-EM structure determination. Nat. Methods 14, 290-296 (2017).

109. Diehl, W. E. et al. Ebola virus glycoprotein with increased infectivity dominated the 2013-2016 epidemic. Cell 167, 1088-1098.e1086 (2016).

\section{Acknowledgements}

We thank Mansun Law and Juan Carlos de la Torre at The Scripps Research Institute and Sujan Shresta at the La Jolla Institute for Immunology for helpful discussions. We thank Christiana Corbaci for creating images used in Figs. 5a, e, and 6a and Michael Arends for proofreading the manuscript. Diffraction data were collected at the Advanced Photon Source (APS) beamline 23-IDD, and Stanford Synchrotron Radiation Lightsource (SSRL) beamline 12-2. Use of the APS was supported by the DOE, Basic Energy Sciences, Office of Science, under contract no. DE-AC02-06CH11357. Use of the SSRL was supported by the US Department of Energy, Basic Energy Sciences, Office of Science, under contract no. DE-AC02-76SF00515. This work was funded in part by NIH grants AI129698 (to J.Z.), AI140844 (to J.Z. and I.A.W.), UfoVax/SFP-2018-0416, and UfoVax/ SFP-2018-1013 (to J.Z.)

\section{Author contributions}

Project design by L.H., A.C., X.L., I.A.W., and J.Z.; rational design of EBOV GPAMuc trimers and nanoparticles by L.H. and J.Z.; plasmid design and processing by L.H. and C. S.; antigen production, purification, and biochemical characterization by A.C., L.H., X.L., E.K., and T.N.; EBOV antibody production by X.L., E.K., and T.N.; negative-stain EM by L.H., T.A., G.O., and A.B.W.; DSC measurement by S.K.; crystallography by A.C. and R. L.S.; BLI by L.H. and J.Z.; mouse plasma-antigen ELISA and rabbit serum-antigen ELISA by L.H. and X.L.; mouse and rabbit IgG purification by L.H. and C.S. ebolavirus-pp and MLV-pp neutralization assays by X.L. and L.H.; antigen-specific mouse B-cell sorting by C.S. and L.H.; mouse B-cell library preparation and NGS by L.H. and C.S.; bioinformatics analysis by L.H. and J.Z.; Manuscript written by L.H., A.C., X.L., I.A.W., and J.Z. All authors were asked to comment on the manuscript. The TSRI manuscript number is 30009 .

\section{Competing interests}

The authors declare no competing interests.

\section{Additional information}

Supplementary information The online version contains supplementary material available at https://doi.org/10.1038/s41467-021-22867-w.

Correspondence and requests for materials should be addressed to I.A.W. or J.Z.

Peer review information Nature Communications thanks Janet Smith and the other anonymous, reviewer(s) for their contribution to the peer review of this work. Peer reviewer reports are available.

Reprints and permission information is available at http://www.nature.com/reprints

Publisher's note Springer Nature remains neutral with regard to jurisdictional claims in published maps and institutional affiliations.

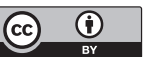

Open Access This article is licensed under a Creative Commons Attribution 4.0 International License, which permits use, sharing, adaptation, distribution and reproduction in any medium or format, as long as you give appropriate credit to the original author(s) and the source, provide a link to the Creative Commons license, and indicate if changes were made. The images or other third party material in this article are included in the article's Creative Commons license, unless indicated otherwise in a credit line to the material. If material is not included in the article's Creative Commons license and your intended use is not permitted by statutory regulation or exceeds the permitted use, you will need to obtain permission directly from the copyright holder. To view a copy of this license, visit http://creativecommons.org/ licenses/by/4.0/

(C) The Author(s) 2021, corrected publication 2022 